\title{
Theoretical Analysis (Convergence and Stability) of a Difference Approximation for Multiterm Time Fractional Convection Diffusion-Wave Equations with Delay
}

\author{
A. S. Hendy ${ }^{1,2}$ and R. H. De Staelen ${ }^{3,4, *(D)}$ \\ 1 Department of Computational Mathematics and Computer Science, Institute of Natural Sciences and \\ Mathematics, Ural Federal University, 19 Mira St., 620002 Yekaterinburg, Russia; \\ ahmed.hendy@fsc.bu.edu.eg \\ 2 Department of Mathematics, Faculty of Science Benha University, Benha 13511, Egypt \\ 3 Department of Electronics and Information Systems, Ghent University, 9000 Gent, Belgium \\ 4 Beheer en Algemene Directie, Ghent University Hospital, C. Heymanslaan 10, 9000 Gent, Belgium \\ * Correspondence: rob.destaelen@ugent.be
}

Received: 31 August 2020; Accepted: 23 September 2020; Published: 03 October 2020

\begin{abstract}
In this paper, we introduce a high order numerical approximation method for convection diffusion wave equations armed with a multiterm time fractional Caputo operator and a nonlinear fixed time delay. A temporal second-order scheme which is behaving linearly is derived and analyzed for the problem under consideration based on a combination of the formula of $L_{2}-1_{\sigma}$ and the order reduction technique. By means of the discrete energy method, convergence and stability of the proposed compact difference scheme are estimated unconditionally. A numerical example is provided to illustrate the theoretical results.
\end{abstract}

Keywords: fractional convection diffusion-wave equations; compact difference scheme; nonlinear delay; spatial variable coefficients; convergence and stability

MSC: 65M06; 35K15; 35K55; 35K57

\section{Introduction}

Fractional derivatives and integrals have recently gained high interest in many fields of science. The ability of classifying and capturing the memory and hereditary properties of various materials and processes is an advantage of fractional derivatives over their integer counterparts, e.g., the modeling of anomalous diffusion by fractional differential equations gives more informative and interesting models [1]. For time-fractional differential equations, the memory feature implies that all previous information is needed to evaluate the time fractional derivative at the current time level. Accordingly, designing a numerical differentiation formula of good accuracy is as ever paramount, but especially hard. The approximation formulas based on the interpolation approximation, such as L1 [2] and $L_{2}-1_{\sigma}$ [3], are of significance to design numerical algorithms to solve time-fractional differential equations. Demonstrated applications in numerous seemingly diverse and widespread fields of physics, such as in porous and glassy materials, in percolation clusters over fractals to semi-conductors, polymers, random media, and beyond, like geophysical and biological systems or processes (e.g., [4,5]), can be effectively modeled by time-fractional diffusion-wave equations of different types. Here, we are seeking to design a compact difference scheme that behaves linearly to numerically solve the non-linear 
delayed multiterm time fractional convection diffusion-wave equation (dmfCDWEs) with spatial variable coefficients. More specifically, we consider

$$
\sum_{r=0}^{m} p_{r} \frac{\partial^{\alpha_{r}} u(x, t)}{\partial t^{\alpha_{r}}}=\frac{\partial}{\partial x}\left(q_{1}(x) \frac{\partial u}{\partial x}\right)+q_{2}(x) \frac{\partial u}{\partial x}+f(u(x, t), u(x, t-s), x, t), 0<t \leq T, 0 \leq x \leq L,
$$

with the following initial and boundary conditions

$$
\begin{aligned}
& u(x, t)=d(x, t), \quad 0 \leq x \leq L, \quad t \in[-s, 0), \quad \frac{\partial u(x, 0)}{\partial t}=\psi(x)=\lim _{t \rightarrow-0} \frac{\partial d(x, t)}{\partial t} \\
& u(0, t)=\phi_{0}(t), \quad u(L, t)=\phi_{L}(t), \quad 0<t \leq T
\end{aligned}
$$

where $s>0$ is a fixed delay parameter, $q_{1}(x)$ and $q_{2}(x)$ are functions chosen, respectively, to be sufficiently and arbitrary differentiable functions. The fractional derivative is defined in Caputo sense and the fractional orders $\left\{\alpha_{r} \mid 1 \leq r \leq m\right\}$ are specified in the manner $\left\{1<\alpha_{m} \leq \alpha_{m-1}<\cdots<\alpha_{0}=2\right\}$. The existence and uniqueness of the global mild solutions for the problem of nonlinear fractional reaction-diffusion equations with delay and Caputo's fractional derivatives are addressed in [6].

This work can be considered to be an extension of our previously published work [7], in which we discussed a single term time fractional wave equation with spatial constant coefficients. The scheme was of $2-\alpha$ order in time and fourth in space. Here, we treat the multiterm time fractional order case with spatial variable coefficients and seek to have temporal second order of convergence. Accordingly, we hinged on the proposed numerical formula in [8] to approximate the multiterm Caputo fractional derivatives of order $\alpha_{r}\left(0<\alpha_{r} \leq 1\right)$ at the super-convergent point. The formula $L_{2}-1_{\sigma}$ can achieve at least second-order accuracy at this point. We rely on the compact operator proposed in [9] in order to attain a fourth order accuracy with respect to space in case of having spatial variable coefficients.

There are some results and findings available regarding the theoretical analysis and numerical computation of single term time fractional sub or super diffusion equations with delay. In [10], the authors introduced a satisfactory numerical method for time fractional diffusion equations with delay. In [11], a novel discrete Grönwall inequality is used to simplify the analysis of difference schemes for time-fractional multi-delayed sub-diffusion equations. Convergence and stability of a compact finite difference method for nonlinear time fractional reaction-diffusion equations with a fixed delay are proposed in [12] by the aid of a new discrete form of the fractional Grönwall inequalities. A numerical solution for a class of time fractional diffusion equations with delay is proposed in [13] that is based on a smooth difference approximation of specific $L 1$ type. Additionally, there are many difference and spectral approaches proposed for multiterm or distributed order time fractional differential equations. An efficient spectral method that is based on Jacobi-Gauss-Radau collocation is applied in order to solve a system of multi-dimensional distributed-order generalized Schrödinger equations in [14]. A combined difference and Galerkin-Legendre spectral method in [15] is used to solve time fractional diffusion equations with nonlinear source term. A Legendre spectral-collocation method for the numerical solution of distributed-order fractional initial value problems is designed in [16]. In [17], the authors proposed a spectral $\tau$-scheme to discretize the fractional diffusion equation with distributed-order fractional derivative in time and Dirichlet boundary conditions. The model solution is expanded in multi-dimensions in terms of Legendre polynomials and the discrete equations are obtained with the $\tau$-method. The two-dimensional distributed-order time fractional cable equation is numerically solved based on the finite difference/spectral method, as clarified in [18]. Two classes of finite difference methods that are based on backward differential formula discretization in the temporal direction are proposed in [19] to efficiently solve the semilinear space fractional reaction-diffusion equation with time-delay. The coefficients of the problem are constants, a fractional centered difference approximation is employed for the space fractional derivative [20], and it gains a fourth order approximation in space due to the use of a specific compact operator [21]. 
The main purpose of our work is the manufacturing of a difference scheme for problems of the kind of (1). Until now, few works hace paid close attention to the multiterm fractional wave equation with variable coefficients and delay argument simultaneously. It is well known that it may be a more challenging task to solve the fractional partial differential equation with delay effectively, since the evolution of a fractional partial differential equation with delay at time $t$ not only depends on its value at $t-s$, but also depends on all previous solutions due to the non-locality of the fractional operator. Higher order numerical schemes are extremely scarce and difficult in regard to the analysis and implementation for the variable coefficient multiterm time fractional convection-diffusion wave equation with delay. For a single temporal term for that kind of problem, we refer to [22]. For the multiterm problem, we design the difference scheme at a super-convergent point to gain a high order of convergence [8] which is more challenging than the single term problem at the level of theoretical analysis.

In order to simplify the problem, an exponential transformation technique [23] can be applied to system (1) to avoid the difficulties resulting from the spatial variable when constructing high order compact difference methods. That technique is used to eliminate the convection term. Assume that $\frac{q_{1}(x)}{q_{2}(x)}$ is integrable in the spatial interval $[0, L]$ and let $u(x, t)=\exp \left(\int_{0}^{x} \tilde{r}(\mathbf{s}) d \mathbf{s}\right) \omega(x, t)$, this yields

$$
\begin{aligned}
\frac{\partial^{\alpha_{r}} u}{\partial t^{\alpha_{r}}} & =\exp \left(\int_{0}^{x} \tilde{r}(\mathbf{s}) d \mathbf{s}\right) \frac{\partial^{\alpha_{r}} \omega}{\partial t^{\alpha_{r}}} \\
\frac{\partial u}{\partial x} & =\exp \left(\int_{0}^{x} \tilde{r}(\mathbf{s}) d \mathbf{s}\right)\left[\tilde{r}(x) \omega(x, t)+\frac{\partial \omega}{\partial x}\right] \\
\frac{\partial^{2} u}{\partial x^{2}} & =\exp \left(\int_{0}^{x} \tilde{r}(\mathbf{s}) d \mathbf{s}\right)\left[\tilde{r}^{2}(x) \omega(x, t)+2 \tilde{r}(x) \frac{\partial \omega}{\partial x}+\tilde{r}^{\prime}(x) \omega(x, t)+\frac{\partial^{2} \omega}{\partial x^{2}}\right] .
\end{aligned}
$$

When substituting (2)-(4) into (1), one directly obtains

$$
\begin{aligned}
\sum_{r=0}^{m} p_{r} \frac{\partial^{\alpha_{r}} \omega(x, t)}{\partial t^{\alpha_{r}}} & =q_{1}(x) \frac{\partial^{2} \omega}{\partial x^{2}}+\left[q_{1}^{\prime}(x)+2 q_{1}(x) \tilde{r}(x)+q_{2}(x)\right] \frac{\partial \omega}{\partial x} \\
& +\left[q_{1}(x) \tilde{r}^{2}(x)+q_{1}(x) \tilde{r}^{\prime}(x)+q_{1}^{\prime}(x) \tilde{r}(x)+q_{2}(x) \tilde{r}(x)\right] \omega(x, t) \\
& +\exp \left(-\int_{0}^{x} \tilde{r}(\mathbf{s}) d \mathbf{s}\right) g(\omega(x, t), \omega(x, t-s), x, t)
\end{aligned}
$$

where, $g(\omega(x, t), \omega(x, t-s), x, t)=f(u(x, t), u(x, t-s), x, t)$. By selecting $\tilde{r}(x)=-\frac{1}{2} \frac{q_{2}(x)}{q_{1}(x)}$, the system (1) is transformed in

$$
\sum_{r=0}^{m} p_{r} \frac{\partial^{\alpha_{r}} \omega(x, t)}{\partial t^{\alpha_{r}}}=\frac{\partial}{\partial x}\left(q_{1}(x) \frac{\partial \omega}{\partial x}\right)+\tilde{f}(\omega(x, t), \omega(x, t-s), x, t), 0<t \leq T, 0 \leq x \leq L,
$$

with the following initial and boundary conditions

$$
\begin{aligned}
& \omega(x, t)=\exp \left(-\int_{0}^{x} \tilde{r}(\mathbf{s}) d \mathbf{s}\right) d(x, t):=\tilde{d}(x, t), \quad t \in[-s, 0), \quad \frac{\partial \omega(x, 0)}{\partial t}=\lim _{t \rightarrow-0} \frac{\partial \tilde{d}(x, t)}{\partial t}:=\tilde{\psi}(x), \\
& \omega(0, t)=\phi_{0}(t), \quad \omega(L, t)=\exp \left(-\int_{0}^{x} \tilde{r}(\mathbf{s}) d \mathbf{s}\right) \phi_{L}(t):=\tilde{\phi}_{L}(t), \quad 0<t \leq T
\end{aligned}
$$

where

$$
\begin{aligned}
\tilde{f}(\omega(x, t), \omega(x, t-s), x, t) & =\left[q_{1}(x) \tilde{r}^{2}+q_{1}(x) \tilde{r}^{\prime}(x)+q_{1}^{\prime}(x) \tilde{r}(x)+q_{2}(x) \tilde{r}(x)\right] \omega(x, t) \\
& +\exp \left(-\int_{0}^{x} \tilde{r}(\mathbf{s}) d \mathbf{s}\right) g(\omega(x, t), \omega(x, t-s), x, t) .
\end{aligned}
$$


In order to transform (5) to a system with zero Dirichlet boundary conditions, we define $h(x, t):=$ $\phi_{0}(t)+\frac{x}{L}\left(\tilde{\phi}_{L}(t)-\phi_{0}(t)\right)$ and introduce the new function $v(x, t)=\omega(x, t)-h(x, t)$. Hence, we have

$$
\sum_{r=0}^{m} p_{r} \frac{\partial^{\alpha_{r}} v(x, t)}{\partial t^{\alpha_{r}}}=\frac{\partial}{\partial x}\left(q_{1}(x) \frac{\partial v}{\partial x}\right)+\tilde{f}(v(x, t), v(x, t-s), x, t), 0<t \leq T, 0 \leq x \leq L
$$

with the following initial and boundary conditions

$$
\begin{aligned}
& v(x, t)=\hat{r}(x, t), \quad 0 \leq x \leq L, \quad t \in[-s, 0), \quad \frac{\partial v(x, 0)}{\partial t}=\hat{\psi}(x)=\lim _{t \rightarrow-0} \frac{\partial r(x, t)}{\partial t} \\
& v(0, t)=v(L, t)=0, \quad t>0 .
\end{aligned}
$$

According to the new transformed system (6), we analytically overcame the first degree of complexity by the elimination of the convection term. There are still two degrees of complexity to be numerically overcome; the multiterm fractional order on the one hand and the nonlinear delay on the other.

Throughout this work, we assume that the function $f(\mu, v, x, t)$ and the solution $v(x, t)$ of $(6)$ are sufficiently smooth in the following sense:

- $\quad$ Assume that $v(x, t) \in \mathrm{C}^{(6,4)}([0, L] \times[0, T])$,

- The partial derivatives $\tilde{f}_{\mu}(\mu, v, x, t)$ and $\tilde{f}_{v}(\mu, v, x, t)$ are continuous in the $\epsilon_{0}$-neighborhood of the solution. Define

$$
\begin{aligned}
c_{1}= & \sup _{\substack{0<x<L, 0<t \leq T \\
\left|\epsilon_{1}\right| \leq \epsilon_{0},\left|\epsilon_{2}\right| \leq \epsilon_{0}}}\left|\tilde{f}_{\mu}\left(v(x, t)+\epsilon_{1}, v(x, t-s)+\epsilon_{2}, x, t\right)\right| \\
c_{2}= & \max _{\substack{0<x<L, 0<t \leq T \\
\left|\epsilon_{1}\right| \leq \epsilon_{0},\left|\epsilon_{2}\right| \leq \epsilon_{0}}}\left|\tilde{f}_{v}\left(u(x, t)+\epsilon_{1}, v(x, t-s)+\epsilon_{2}, x, t\right)\right| .
\end{aligned}
$$

The structure of this paper is arranged, as follows. First, we introduce a derivation of the compact difference scheme. Next, in the third section, convergence and stability for the compact difference scheme are carried out. Finally, the paper ends with a numerical illustration and conclusion.

\section{A Compact Difference Scheme}

A linearized numerical method that combines the super-convergence approximation $L_{2}-1_{\sigma}$ with the order reduction method is derived. Some further notations are fixed before we continue. Take two positive integers $M$ and $n_{0}$, let $h=\frac{L}{M}, \tau=\frac{s}{n_{0}}$ and denote $x_{i}=i h$ for $i=0, \ldots, M ; t_{k}=k \tau$ and $t_{k+\sigma}=(k+\sigma) \tau$, for $k=-n_{0}, \ldots, N$, where $N=\left\lfloor\frac{T}{\tau}\right\rfloor$. Using the points $x_{i}$ in space and $t_{k}$ in time, we cover the space-time domain by $\Omega_{h \tau}=\Omega_{h} \times \Omega_{\tau}$, where $\Omega_{h}=\left\{x_{i} \mid 0 \leq i \leq M\right\}$ and $\Omega_{\tau}=\left\{t_{k} \mid-n_{0} \leq k \leq N\right\}$.

\section{1. $L_{2}-1_{\sigma}$ Super-Convergence Scheme}

Here, we give a preliminary for the Alikhanov scheme. Denote $\gamma_{r}=\alpha_{r}-1(0 \leq r \leq m)$ and

$$
\mathcal{F}(\sigma)=\sum_{r=0}^{m} \frac{p_{r}}{\Gamma\left(3-\gamma_{r}\right)} \sigma^{1-\gamma_{r}}\left[\sigma-\left(1-\frac{\gamma_{r}}{2}\right)\right] \tau^{2-\gamma_{r}}
$$

such that $0<\gamma_{m}<\gamma_{m-1}<\cdots<\gamma_{0} \leq 1$. Additionally, now we invoke the following lemmas from Alikhanov work.

Lemma 2.1 ([8]). A unique positive root $\sigma^{*} \in\left[1-\gamma_{0} / 2,1-\gamma_{m} / 2\right]$ exists for $\mathcal{F}(\sigma)=0$. 
Lemma 2.2 ([8]). For $m=0$, the root of $\mathcal{F}(\sigma)=0$ is $1-\gamma_{0} / 2$. However, if $m \geq 1$, the root $\sigma^{*}$ can be obtained by the Newton iteration method. The Newton iteration sequence $\left\{\sigma_{k}\right\}_{k=0}^{\infty}$ generated by $\sigma_{0}=1-\gamma_{m} / 2$ and $\sigma_{k+1}=\sigma_{k}-\frac{\mathcal{F}\left(\sigma_{k}\right)}{\mathcal{F}^{\prime}\left(\sigma_{k}\right)}$ for $k=0,1,2, \ldots$ is monotonically decreasing and convergent to $\sigma^{*}$.

For the sake of simplicity, let $\sigma=\sigma^{*}$ here and later. Define [3]

$$
\begin{gathered}
a_{0}=\sigma^{1-\gamma}, \quad a_{l}^{\gamma}=(\sigma+l)^{1-\gamma}-(\sigma+l-1)^{1-\gamma}, \\
b_{l}^{\gamma}=\frac{1}{2-\gamma}\left[(l+\sigma)^{2-\gamma}-(l-1+\sigma)^{2-\gamma}\right]-\frac{1}{2}\left[(l+\sigma)^{1-\gamma}+(l-1+\sigma)^{1-\gamma}\right],
\end{gathered}
$$

for each $l \in \mathbb{N}^{+}$. Next, we define $\left\{C_{n}^{(k+1, \gamma)}\right\}$, as follows

$$
C_{n}^{(k+1, \gamma)}= \begin{cases}a_{0}, & n=0, k=0 \\ a_{0}^{\gamma}+b_{1}^{\gamma}, & n=0, k \geq 1 \\ a_{n}^{\gamma}+b_{n+1}^{\gamma}-b_{n}^{\gamma}, & 1 \leq n \leq k-1, k \geq 1, \\ a_{k}^{\gamma}-b_{k}^{\gamma}, & n=k, k \geq 1\end{cases}
$$

Denote

$$
\hat{C}_{n}^{(k+1)}=\sum_{r=0}^{m} p_{r} \frac{\tau^{-\gamma_{r}}}{\Gamma\left(2-\gamma_{r}\right)} C_{n}^{\left(k+1, \gamma_{r}\right)}, \quad \hat{b}_{n}=\sum_{r=0}^{m} p_{r} \frac{\tau^{-\gamma_{r}}}{\Gamma\left(2-\gamma_{r}\right)} b_{n}^{\left(\gamma_{r}\right)}, \quad n=0,1, \ldots, k
$$

The next two lemmas are devoted to the properties of the coefficients $\hat{C}_{n}^{(k)}$ and $\hat{b}_{n}$.

Lemma 2.3 ([8]). Given any non-negative integer $m$ and positive constants $p_{0}, p_{1}, \ldots, p_{m}$, for any $\left\{\gamma_{r} \in\right.$ $(0,1] \mid r=0,1, \cdots, m\}$ it holds

$$
\hat{C}_{1}^{(k+1)}>\hat{C}_{2}^{(k+1)}>\cdots>\hat{C}_{k-2}^{(k+1)}>\hat{C}_{k-1}^{(k+1)}>\sum_{r=0}^{m} p_{r} \frac{\tau^{-\gamma_{r}}}{\Gamma\left(2-\gamma_{r}\right)} \frac{1-\gamma_{r}}{2}(k-1+\sigma)^{-\gamma_{r}}
$$

In addition, there exists a $\tau_{0}>0$, such that $(2 \sigma-1) \hat{C}_{0}^{(k+1)}-\sigma \hat{C}_{1}^{(k+1)}>0$, when $\tau \leq \tau_{0}, n=2,3, \cdots$, and $\hat{C}_{0}^{(k+1)}>\hat{C}_{1}^{(k+1)}$.

Lemma 2.4 ([24]). The sequences $\hat{C}_{n}^{(k)}$ and $\hat{b}_{n}$ satisfy

$$
\hat{C}_{n}^{(k+1)}= \begin{cases}C_{n}^{(k)}, & 0 \leq n \leq k-2 \\ C_{n}^{(k)}+\hat{b}_{n+1}, & n=k-1\end{cases}
$$

Additionally, the following estimates hold:

$$
\sum_{n=1}^{k} \hat{C}_{n}^{(k+1)} \leq \sum_{r=0}^{m} p_{r} \frac{3 \tau^{-\gamma_{r}}}{2 \Gamma\left(2-\gamma_{r}\right)}(k+\sigma)^{1-\gamma_{r}}
$$

and

$$
\sum_{n=1}^{k} \hat{b}_{n} \leq \sum_{r=0}^{m} p_{r} \frac{\gamma_{r} \tau^{-\gamma_{r}}}{2 \Gamma\left(3-\gamma_{r}\right)}(k+\sigma)^{1-\gamma_{r}}
$$

Let $\mathcal{W}_{h}=\left\{w: \Omega_{h \tau} \rightarrow \mathbb{R} \mid w\left(x_{i}, t_{k}\right)=w_{i}^{k} ; i=0,1, \ldots, M ; k=-n_{0},-n_{0}+1, \ldots, N\right\}$ be a grid function space on $\Omega_{h \tau}$ and also define $\hat{\mathcal{W}}_{h}=\left\{w \in \mathcal{W}_{h}, w\left(x_{0}, \cdot\right)=w\left(x_{M}, \cdot\right)=0\right\}$. Introduce the following notations 


$$
\begin{aligned}
w_{i+1 / 2}^{k} & =\frac{1}{2}\left[w_{i+1}^{k}+w_{i}^{k}\right], \quad \delta_{x} w_{i+1 / 2}^{k}=\frac{1}{h}\left[w_{i+1}^{k}-w_{i}^{k}\right], \quad \delta_{t} w_{i}^{\frac{1}{2}}=\frac{1}{\tau}\left[w_{i}^{1}-w_{i}^{0}\right], \\
\delta_{\hat{x}} w_{i}^{k} & =\frac{1}{2 h}\left[w_{i+1}^{k}-w_{i-1}^{k}\right], \quad \delta_{x}^{2} w_{i}^{k}=\frac{1}{h}\left[\delta_{x} w_{i+1 / 2}^{k}-\delta_{x} w_{i-1 / 2}^{k}\right], \\
w_{i}^{k+\sigma} & =\sigma w_{i}^{k+1}+(1-\sigma) w_{i}^{k}, \quad \partial_{\hat{t}} w_{i}^{k}=\frac{1}{2 \tau}\left[(2 \sigma+1) w_{i}^{k+1}-4 \sigma w_{i}^{k}+(2 \sigma-1) w_{i}^{k-1}\right] .
\end{aligned}
$$

Moreover, denote $\varkappa_{1}=\left(q_{1}^{\prime}\right)^{2} / q_{1}-\frac{1}{2} q_{1}^{\prime \prime}$ and $\varkappa_{2}=q_{1}-\frac{h^{2}}{12} \varkappa_{1}$. The compact operator acting on the spatial variable is defined as [9],

$$
\mathcal{A} w_{i}= \begin{cases}w_{i}+\frac{h^{2}}{12}\left[\delta_{x}^{2} w_{i}-\delta_{\hat{x}}\left(\frac{q_{1}^{\prime}}{q_{1}} w\right)_{i}\right], & i=1, \ldots, M-1, \\ w_{i}, & i=0, M .\end{cases}
$$

Lemma 2.5 ([9]). Let $g(x) \in C^{6}[0, L]$, such that $f(x)=\partial_{x}\left(q_{1}(x) g(x)\right)$, and then it holds

$$
\mathcal{A} F_{i}=\delta_{x}\left(\varkappa_{2}(x) \delta_{x} G\right)_{i}+\mathrm{O}\left(h^{4}\right),
$$

where $F_{i}=f\left(x_{i}\right)$ and $G_{i}=g\left(x_{i}\right)$.

For any $v, w \in \mathcal{W}_{h}$, we define the following inner products

$$
\begin{gathered}
\langle v, w\rangle=h\left[\frac{1}{2} v_{0} w_{0}+\sum_{i=1}^{M-1} v_{i} w_{i}+\frac{1}{2} v_{M} w_{M}\right], \quad\langle v, w\rangle_{1}=h \sum_{i=1}^{M-1}\left(\delta_{x} w_{i+1 / 2}\right)\left(\delta_{x} v_{i+1 / 2}\right), \\
\langle v, w\rangle_{\varkappa_{2}}=h \sum_{i=1}^{M-1} \varkappa_{2}\left(x_{i+1 / 2}\right)\left(\delta_{x} w_{i+1 / 2}\right)\left(\delta_{x} v_{i+1 / 2}\right), \quad\langle w, v\rangle_{\mathcal{A}}=\langle w, v\rangle-\frac{h^{2}}{12}\langle w, v\rangle .
\end{gathered}
$$

and their corresponding norms and semi-norms

$$
\|w\|^{2}=\langle w, w\rangle, \quad|w|_{1}^{2}=\langle w, w\rangle_{1}, \quad\|w\|_{\mathcal{A}}^{2}=\langle w, w\rangle_{\mathcal{A}}, \quad|w|_{1, \varkappa_{2}}^{2}=\langle w, w\rangle_{\varkappa_{2}}, \quad\|w\|_{\infty}=\max _{0 \leq i \leq M}\left|w_{i}\right| .
$$

Furthermore, assume that the coefficients satisfy

$$
b_{0} \leq q_{1}(x) \leq b_{1}, \quad b_{2} \leq \varkappa_{2}(x) \leq b_{3}, \quad\left|\frac{q_{1}^{\prime}}{q_{1}}\right| \leq b_{3}
$$

where all $b_{i}$ are positive constants.

Lemma 2.6 ([25]). For any grid function $w \in \hat{\mathcal{W}}_{h}$, it holds that

$$
\|w\| \leq \frac{L}{\sqrt{6}}|w|_{1}, \quad\|w\|_{\infty} \leq \frac{\sqrt{L}}{2}|w|_{1}, \quad \sqrt{\frac{2}{3}}\|w\| \leq\|w\|_{\mathcal{A}} \leq\|w\| .
$$

Lemma 2.7 ([26]). For any grid function $w_{1}, w_{2} \in \hat{\mathcal{W}}_{h}$, it holds that

$$
|\langle\mathcal{A} w, w\rangle| \geq\|w\|_{\mathcal{A}}^{2}-\frac{c_{3} h}{12}\|w\|^{2}, \quad\left|\left\langle\mathcal{A} w_{1}, w_{2}\right\rangle\right| \leq \frac{1}{2}\left[\left\|w_{1}\right\|_{\mathcal{A}}^{2}+\left\|w_{2}\right\|_{\mathcal{A}}^{2}\right]+\frac{c_{3} h}{24}\left[\left\|w_{1}\right\|^{2}+\left\|w_{2}\right\|^{2}\right] .
$$

Lemma 2.8 ([8]). For any $h(t) \in C^{3}[0, T]$ and $\gamma_{r} \in(0,1], 0 \leq r \leq m$, such that $\gamma_{0}>\gamma_{1}>\cdots>\gamma_{m \prime \prime}$ the $L_{2}-1_{\sigma}$ formula has the following order of convergence 


$$
\begin{aligned}
\sum_{r=0}^{m} p_{r} D_{t}^{\gamma_{r}} h\left(t_{k+\sigma}\right) & =\sum_{n=0}^{k}\left(\sum_{r=0}^{m} p_{r} \frac{\tau^{-\gamma_{r}}}{\Gamma\left(2-\gamma_{r}\right)} C_{n}^{\left(k+1, \gamma_{r}\right)}\right)\left(h\left(t_{k-n+1}\right)-h\left(t_{k-n}\right)\right)+\mathcal{O}\left(\tau^{3-\gamma_{0}}\right) \\
& =\sum_{n=0}^{k} \hat{C}_{k-n}^{(k+1)}\left[h\left(t_{n+1}\right)-h\left(t_{n}\right)\right]+\mathcal{O}\left(\tau^{3-\gamma_{0}}\right) .
\end{aligned}
$$

Lemma 2.9 ([24]). For any $h(t) \in C^{3}[0, T]$, we can obtain

$$
\begin{aligned}
\partial_{\hat{t}} h\left(t_{k}\right) & \cong \frac{1}{2 \tau}\left[(2 \sigma+1) h\left(t_{k+1}\right)-4 \sigma h\left(t_{k}\right)+(2 \sigma-1) h\left(t_{k-1}\right)\right] \\
& =\frac{\partial h}{\partial t}\left(t_{k+\sigma}\right)+\mathcal{O}\left(\tau^{2}\right), \quad k \geq 1
\end{aligned}
$$

Lemma 2.10 ([24]). Suppose that $\langle\cdot, \cdot\rangle_{*}$ is an inner product on $\hat{\mathcal{W}}_{h}$ and $\|\cdot\|_{*}$ is a norm deduced by the inner product. For any grid functions $w_{0}, w_{1}, \ldots, w_{k+1} \in \hat{\mathcal{W}}_{h}$, we have the following inequality

$$
\begin{gathered}
\left\langle\sum_{n=0}^{k} \hat{C}_{k-n}^{(k+1)}\left[w^{n+1}-w^{n}\right], w^{k+\sigma}\right\rangle_{*} \geq \frac{1}{2} \sum_{n=0}^{k} \hat{C}_{k-n}^{(k+1)}\left[\left\|w^{n+1}\right\|_{*}^{2}-\left\|w^{n}\right\|_{*}^{2}\right], \\
\left\langle\partial_{\hat{t}} w^{k}, w^{k+\sigma}\right\rangle_{*} \geq \frac{1}{4 \tau}\left(\mathcal{E}^{k+1}-\mathcal{E}^{k}\right),
\end{gathered}
$$

where

$$
\mathcal{E}^{k+1}=(2 \sigma+1)\left\|w^{k+1}\right\|^{2}-(2 \sigma-1)\left\|w^{k}\right\|^{2}+\left(2 \sigma^{2}+\sigma-1\right)\left\|w^{k+1}-w^{k}\right\|^{2}, \quad k \geq 0 .
$$

Additionally, it holds that

$$
\mathcal{E}^{k+1} \geq \frac{1}{\sigma}\left\|w^{k+1}\right\|^{2}, \quad k \geq 0 .
$$

Let us initiate by order reduction for system (6) by letting $\gamma_{r}=\alpha_{r}-1,(0 \leq r \leq M)$ and $\mathcal{V}(x, t)=v_{t}(x, t)$, then

$$
\frac{\partial^{\alpha_{r}} v(x, t)}{\partial t^{\alpha_{r}}}=\frac{\partial^{\gamma_{r}} \mathcal{V}(x, t)}{\partial t^{\gamma_{r}}}
$$

and so

$$
\partial_{t}\left(\frac{\partial}{\partial x}\left(q_{1}(x) \frac{\partial v}{\partial x}\right)\right)=\frac{\partial}{\partial x}\left(q_{1}(x) \frac{\partial \mathcal{V}}{\partial x}\right) .
$$

Subsequently, the equivalent system to (6) after order reduction can be formulated as

$$
\begin{aligned}
\sum_{r=0}^{m} p_{r} \frac{\partial^{\gamma_{r}} \mathcal{V}(x, t)}{\partial t \gamma_{r}} & =\frac{\partial}{\partial x}\left(q_{1}(x) \frac{\partial \nu}{\partial x}\right)+\tilde{f}(v(x, t), v(x, t-s), x, t), 0<t \leq T, 0 \leq x \leq L, \\
\partial_{t}\left(\frac{\partial}{\partial x}\left(q_{1}(x) \frac{\partial \nu}{\partial x}\right)\right) & =\frac{\partial}{\partial x}\left(q_{1}(x) \frac{\partial \mathcal{V}}{\partial x}\right),
\end{aligned}
$$

with the following initial and boundary conditions

$$
\begin{aligned}
& v(x, t)=\hat{r}(x, t), \quad 0 \leq x \leq L, \quad t \in[-s, 0), \quad \mathcal{V}(x, 0)=\hat{\psi}(x), \quad 0 \leq x \leq L, \\
& v(0, t)=v(L, t)=0, \quad 0<t \leq T, \\
& \mathcal{V}(0, t)=\mathcal{V}(L, t)=0, \quad 0<t \leq T .
\end{aligned}
$$

\subsection{Compact Difference Scheme Construction}

Suppose that $v_{t}(x, t)=\mathcal{V}(x, t) \in \mathbf{C}_{x, t}^{6,4}([0, T] \times[0, L])$, define the discretized functions

$$
\mathbf{V}_{i}^{k}=\mathcal{V}\left(x_{i}, t_{k}\right), \quad \mathrm{V}_{i}^{k}=v\left(x_{i}, t_{k}\right), 0 \leq i \leq M, 0 \leq k \leq N .
$$


Consider (20a) at $\left(x_{i}, t_{k+\sigma}\right)$, , and then we get

$$
\sum_{r=0}^{m} p_{r} \frac{\partial^{\gamma_{r}} \mathcal{V}\left(x_{i}, t_{k+\sigma}\right)}{\partial t_{r}}=\frac{\partial}{\partial x}\left(q_{1}\left(x_{i}\right) \frac{\partial v\left(x_{i}, t_{k+\sigma}\right)}{\partial x}\right)+\tilde{f}_{i}^{k+\sigma} 0<i<M, 0 \leq k \leq N-1,
$$

such that

$$
\tilde{f}_{i}^{k}=\tilde{f}\left(v\left(x_{i}, t_{k}\right), v\left(x_{i}, t_{k}-s\right), x_{i}, t_{k}\right) .
$$

From Lemma 2.10, we conclude that

$$
\sum_{r=0}^{m} p_{r} \frac{\partial \gamma_{r} \mathcal{V}\left(x_{i}, t_{k+\sigma}\right)}{\partial t \gamma_{r}}=\sum_{n=0}^{k} \hat{C}_{k-n}^{k+1}\left(\mathbf{V}_{i}^{n+1}-\mathbf{V}_{i}^{n}\right)+\mathrm{O}\left(\tau^{3-\gamma_{0}}\right), \quad 0<i<M, 0 \leq k \leq N-1,
$$

and a direct expansion of Taylor type yields

$$
\tilde{f}_{i}^{k+\sigma}=\tilde{F}_{i}^{k+\sigma}+\mathrm{O}\left(\tau^{2}\right),
$$

such that

$$
\tilde{F}_{i}^{k+\sigma}=\tilde{f}\left((\sigma+1) \mathrm{V}_{i}^{k}-\sigma \mathrm{V}_{i}^{k-1}, \sigma \mathrm{V}_{i}^{k+1-n_{0}}+(1-\sigma) \mathrm{V}_{i}^{k-n_{0}}, x_{i}, t_{k+\sigma}\right) .
$$

Acting the averaging operator $\mathcal{A}$ on both sides of (21), noticing Lemma 2.10 and using Taylor expansion, we arrive at

$$
\sum_{n=0}^{k} \hat{C}_{k-n}^{k+1}\left(\mathcal{A} \mathbf{V}_{i}^{n+1}-\mathcal{A} \mathbf{V}_{i}^{n}\right)=\mathcal{A}\left[\frac{\partial}{\partial x}\left(q_{1}\left(x_{i}\right) \frac{\partial v\left(x_{i}, t_{k+\sigma}\right)}{\partial x}\right)\right]+\mathcal{A} \tilde{f}_{i}^{k+\sigma} 0<i<M, 0 \leq k \leq N-1,
$$

Next, by using Lemma 2.5 and (23), we obtain

$$
\sum_{n=0}^{k} \hat{C}_{k-n}^{k+1}\left(\mathcal{A} \mathbf{V}_{i}^{n+1}-\mathcal{A} \mathbf{V}_{i}^{n}\right)=\delta_{x}\left(\varkappa_{2} \delta_{x} \mathrm{~V}\right)_{i}^{k+\sigma}+\mathcal{A} \tilde{F}_{i}^{k+\sigma}+\mathcal{S}_{i}^{k+\sigma},
$$

where a constant $\tilde{c}_{0}$ exists in order that

$$
\left|\mathcal{S}_{i}^{k+\sigma}\right| \leq \tilde{c}_{0}\left(\tau^{2}+h^{4}\right), \quad 1 \leq i \leq M-1, \quad 0 \leq k \leq N-1 .
$$

By considering (20b) at $\left(x_{i}, t_{1 / 2}\right)$ and $\left(x_{i}, t_{k+\sigma}\right)$, respectively, operating by $\mathcal{A}$ on both equations, we obtain by the aids of Taylor expansions and Lemmas 2.5 and 2.9, which

$$
\begin{aligned}
\delta_{t}\left(\delta_{x}\left(\varkappa_{2} \delta_{x} \mathrm{~V}\right)_{i}^{1 / 2}\right) & =\delta_{x}\left(\varkappa_{2} \delta_{x} \mathbf{V}\right)_{i}^{1 / 2}+s_{i}^{1 / 2}, \quad 1 \leq i \leq M-1, \\
\partial_{\hat{t}}\left(\delta_{x}\left(\varkappa_{2} \delta_{x} \mathrm{~V}\right)_{i}^{k+\sigma}\right) & =\delta_{x}\left(\varkappa_{2} \delta_{x} \mathbf{V}\right)_{i}^{k+\sigma}+s_{i}^{k+\sigma}, \quad 1 \leq i \leq M-1, \quad 1 \leq k \leq N-1 .
\end{aligned}
$$

Moreover, there exists a constant $\tilde{c}_{1}>0$, such that

$$
\begin{aligned}
\left|s_{i}^{1 / 2}\right| \leq \tilde{c}_{1}\left(\tau^{2}+h^{4}\right), & 1 \leq i \leq M-1, \\
\left|s_{i}^{k+\sigma}\right| \leq \tilde{c}_{1}\left(\tau^{2}+h^{4}\right), & 1 \leq i \leq M-1, \quad 1 \leq k \leq N-1 .
\end{aligned}
$$

By omitting the small terms in (26)-(28) and noticing the initial and boundary conditions, we construct a spatial fourth order difference scheme for problem (6), as follows 


$$
\begin{aligned}
\sum_{n=0}^{k} \hat{C}_{k-n}^{k+1}\left(\mathcal{A} \mathcal{V}_{i}^{n+1}-\mathcal{A} \mathcal{V}_{i}^{n}\right) & =\delta_{x}\left(\varkappa_{2} \delta_{x} v\right)_{i}^{k+\sigma}+\mathcal{A} \tilde{\mathcal{F}}_{i}^{k+\sigma}, \quad 1 \leq i \leq M-1, \quad 0 \leq k \leq N-1, \\
\delta_{t}\left(\delta_{x}\left(\varkappa_{2} \delta_{x} v\right)_{i}^{1 / 2}\right) & =\delta_{x}\left(\varkappa_{2} \delta_{x} \mathcal{V}\right)_{i}^{1 / 2}, \quad 1 \leq i \leq M-1, \\
\partial_{\hat{t}}\left(\delta_{x}\left(\varkappa_{2} \delta_{x} v\right)_{i}^{k+\sigma}\right) & =\delta_{x}\left(\varkappa_{2} \delta_{x} \mathcal{V}\right)_{i}^{k+\sigma}, \quad 1 \leq i \leq M-1, \quad 1 \leq k \leq N-1, \\
v_{i}^{k} & =\hat{\mathcal{F}}_{i}^{k}, \quad 1 \leq i \leq M-1, \quad-n_{0} \leq k \leq 0, \quad \mathcal{V}_{i}^{0}=\hat{\psi}\left(x_{i}\right), \quad 1 \leq i \leq M-1, \\
v_{0}^{k} & =v_{M}^{k}=0, \quad 0 \leq k \leq N, \\
\mathcal{V}_{0}^{k} & =\mathcal{V}_{M}^{k}=0, \quad 0 \leq k \leq N .
\end{aligned}
$$

where

$$
\tilde{\mathcal{F}}_{i}^{k+\sigma}=\tilde{f}\left((\sigma+1) v_{i}^{k}-\sigma v_{i}^{k-1}, \sigma v_{i}^{k+1-n_{0}}+(1-\sigma) v_{i}^{k-n_{0}}, x_{i}, t_{k+\sigma}\right) .
$$

\section{The Stability and Convergence of the Constructed Difference Schemes}

First, we will start with some technical lemmas, which will be helpful in the context of convergence and stability.

The nonlinear delay term $\tilde{f}(\mu, v, x, t)$ is sufficiently smooth and it satisfies the Lipschitz condition

$$
\begin{aligned}
\left|\tilde{f}\left(\mu_{1}, v, x, t\right)-\tilde{f}\left(\mu_{2}, v, x, t\right)\right| \leq c_{1}\left|\mu_{1}-\mu_{2}\right|, \quad \forall \mu_{1}, \mu_{2} \in[0, L] \times[0, T], \\
\left|\tilde{f}\left(\mu, v_{1}, x, t\right)-\tilde{f}\left(\mu, v_{2}, x, t\right)\right| \leq c_{1}\left|v_{1}-v_{2}\right|, \quad \forall v_{1}, v_{2} \in[0, L] \times[-\tau, T],
\end{aligned}
$$

where $c_{1}$ and $c_{2}$ are two positive constants.

Lemma 3.1. For any $\mathrm{V}_{i}^{k}, \mathrm{U}_{i}^{k} \in \hat{\mathcal{W}}_{h}$, if we define $\varepsilon_{i}^{k}=\mathrm{V}_{i}^{k}-\mathrm{U}_{i}^{k}$, and also

$$
\tilde{\mathcal{F}}_{i}^{k+\sigma}=\tilde{f}\left((\sigma+1) \mathrm{U}_{i}^{k}-\sigma \mathrm{U}_{i}^{k-1}, \sigma \mathrm{U}_{i}^{k+1-n_{0}}+(1-\sigma) \mathrm{U}_{i}^{k-n_{0}}, x_{i}, t_{k+\sigma}\right),
$$

the following estimate is satisfied

$$
\left\|\tilde{F}_{i}^{k+\sigma}-\tilde{\mathcal{F}}_{i}^{k+\sigma}\right\|^{2} \leq 4\left[2 c_{1}^{2}\left(\left\|\varepsilon_{i}^{k}\right\|^{2}+\left\|\varepsilon_{i}^{k-1}\right\|^{2}\right)+c_{2}^{2}\left(\left\|\varepsilon_{i}^{k+1-n_{0}}\right\|^{2}+\left\|\varepsilon_{i}^{k-n_{0}}\right\|^{2}\right)\right] .
$$

Proof. Recalling $\tilde{F}_{i}^{k+\sigma}$ from (24) and under the assumptions of the nonlinear delay term (33), we can deduce the following estimate

$$
\left|\tilde{F}_{i}^{k+\sigma}-\tilde{\mathcal{F}}_{i}^{k+\sigma}\right| \leq c_{1}\left|(\sigma+1) \varepsilon_{i}^{k}-\sigma \varepsilon_{i}^{k-1}\right|+c_{2}\left|\sigma \varepsilon_{i}^{k+1-n_{0}}+(1-\sigma) \varepsilon_{i}^{k-n_{0}}\right|, \quad 1 \leq i \leq M-1,
$$

and so

$$
\begin{aligned}
\left\|\tilde{F}_{i}^{k+\sigma}-\tilde{\mathcal{F}}_{i}^{k+\sigma}\right\|^{2} & \leq h \sum_{i=1}^{M-1}\left(c_{1}\left|(\sigma+1) \varepsilon_{i}^{k}-\sigma \varepsilon_{i}^{k-1}\right|+c_{2}\left|\sigma \varepsilon_{i}^{k+1-n_{0}}+(1-\sigma) \varepsilon_{i}^{k-n_{0}}\right|\right)^{2} \\
& \leq 2 c_{1}^{2} h \sum_{i=1}^{M-1}\left[\left|(\sigma+1) \varepsilon_{i}^{k}-\sigma \varepsilon_{i}^{k-1}\right|\right]^{2}+2 c_{2}^{2} h \sum_{i=1}^{M-1}\left[\left|\sigma \varepsilon_{i}^{k+1-n_{0}}+(1-\sigma) \varepsilon_{i}^{k-n_{0}}\right|\right]^{2} \\
& \leq 4\left[2 c_{1}^{2}\left(\left\|\varepsilon_{i}^{k}\right\|^{2}+\left\|\varepsilon_{i}^{k-1}\right\|^{2}\right)+c_{2}^{2}\left(\left\|\varepsilon_{i}^{k+1-n_{0}}\right\|^{2}+\left\|\varepsilon_{i}^{k-n_{0}}\right\|^{2}\right)\right]
\end{aligned}
$$

For the convenience of our analysis, the following Grönwall inequality is recalled. 
Lemma 3.2. Suppose that $\left\{H^{k} \mid k \geq 0\right\}$ is a non negative sequence that satisfies

$$
H^{k+1} \leq A+B \tau \sum_{j=1}^{k} H^{j}, \quad k=0,1, \ldots
$$

then

$$
H^{k+1} \leq A \exp (B k \tau), \quad k=0,1, \ldots,
$$

in which the positivity of the constants $A$ and $B$ must be taken into account.

Lemma 3.3. For any $p_{i}^{k}, q_{i}^{k} \in \hat{\mathcal{W}}_{h}$, the following estimates are satisfied

$$
\begin{aligned}
\left\langle\delta_{t}\left(\delta_{x}\left(\varkappa_{2} \delta_{x} p\right)_{i}^{1 / 2}\right),-2 \sigma p_{i}^{1}\right\rangle & =\frac{2 \sigma}{\tau}\left[\left|p_{i}^{1}\right|_{1, \varkappa_{2}}^{2}-\left\langle p_{i}^{0}, p_{i}^{1}\right\rangle_{\varkappa_{2}}\right], \\
\left\langle\delta_{x}\left(\varkappa_{2} \delta_{x} q\right)_{i}^{1 / 2},-2 \sigma p_{i}^{1}\right\rangle & =\sigma\left[\left\langle q_{i}^{1}, p_{i}^{1}\right\rangle_{\varkappa_{2}}+\left\langle q_{i}^{0}, p_{i}^{1}\right\rangle_{\varkappa_{2}}\right], \\
\left\langle\partial_{\hat{t}}\left(\delta_{x}\left(\varkappa_{2} \delta_{x} p\right)_{i}^{k+\sigma}\right),-p_{i}^{k+\sigma}\right\rangle & \geq \frac{1}{4 \tau}\left(\tilde{\mathcal{E}}^{k+1}-\tilde{\mathcal{E}}^{k}\right), \\
\left\langle\delta_{x}\left(\varkappa_{2} \delta_{x} q\right)_{i}^{k+\sigma},-p_{i}^{k+\sigma}\right\rangle & =\left\langle q_{i}^{k+\sigma}, p_{i}^{k+\sigma}\right\rangle_{\varkappa_{2}} .
\end{aligned}
$$

where

$$
\tilde{\mathcal{E}}^{k+1}=(2 \sigma+1)\left|w^{k+1}\right|_{1, \varkappa_{2}}^{2}-(2 \sigma-1)\left|w^{k}\right|_{1, \varkappa_{2}}^{2}+\left(2 \sigma^{2}+\sigma-1\right)\left|w^{k+1}-w^{k}\right|_{1, \varkappa_{2}}^{2} \quad k \geq 0 .
$$

Proof. Starting from the 1.h.s of (40),

$$
\begin{aligned}
\left\langle\delta_{t}\left(\delta_{x}\left(\varkappa_{2} \delta_{x} p\right)_{i}^{1 / 2}\right),-2 \sigma p_{i}^{1}\right\rangle & =\frac{-2 \sigma}{\tau}\left\langle\delta_{x}\left(\left(\varkappa_{2} \delta_{x} p\right)_{i}^{1}\right)-\delta_{x}\left(\left(\varkappa_{2} \delta_{x} p\right)_{i}^{0}\right), p_{i}^{1}\right\rangle \\
& =\frac{2 \sigma}{\tau} h \sum_{i=0}^{M-1}\left[\left(\varkappa_{2}\right)_{i+1 / 2} \delta_{x} p_{i+1 / 2}^{1} \delta_{x} p_{i+1 / 2}^{1}-\left(\varkappa_{2}\right)_{i+1 / 2} \delta_{x} p_{i+1 / 2}^{0} \delta_{x} p_{i+1 / 2}^{1}\right]
\end{aligned}
$$

then the r.h.s of (40) is achieved directly. Additionally, starting from the 1.h.s of (41),

$$
\begin{aligned}
\left\langle\delta_{x}\left(\varkappa_{2} \delta_{x} q\right)_{i}^{1 / 2},-2 \sigma p_{i}^{1}\right\rangle & =\frac{-2 \sigma}{2}\left\langle\delta_{x}\left(\left(\varkappa_{2} \delta_{x} q\right)_{i}^{1}\right)+\delta_{x}\left(\left(\varkappa_{2} \delta_{x} q\right)_{i}^{0}\right), p_{i}^{1}\right\rangle \\
& =\sigma h \sum_{i=0}^{M-1}\left[\left(\varkappa_{2}\right)_{i+1 / 2} \delta_{x} q_{i+1 / 2}^{1} \delta_{x} p_{i+1 / 2}^{1}+\left(\varkappa_{2}\right)_{i+1 / 2} \delta_{x} q_{i+1 / 2}^{0} \delta_{x} p_{i+1 / 2}^{1}\right]
\end{aligned}
$$

then the r.h.s of (41) is immediately held. Invoking the previous estimates and Lemma 2.10, we deduce

$$
\begin{aligned}
\left\langle\partial_{\hat{t}}\left(\delta_{x}\left(\varkappa_{2} \delta_{x} p\right)_{i}^{k+\sigma}\right),-p_{i}^{k+\sigma}\right\rangle & =\left\langle\partial_{\hat{t}} p_{i}^{k+\sigma}, p_{i}^{k+\sigma}\right\rangle_{\varkappa_{2}} \\
& \geq \frac{1}{4 \tau}\left(\tilde{\mathcal{E}}^{k+1}-\tilde{\mathcal{E}}^{k}\right),
\end{aligned}
$$

where $\tilde{\mathcal{E}}^{k+1}$ is defined by (44) and so (42) is achieved. The estimate (43) is simply calculated. 
Now, we are in a position to combine the Lemmas 3.1-3.3 to prove the convergence and stability of the proposed compact difference scheme. To that end, Let

$$
\rho_{i}^{k}=\mathbf{V}_{i}^{k}-\mathcal{V}_{i}^{k}, \quad e_{i}^{k}=\mathrm{V}_{i}^{k}-v_{i}^{k}, \quad 0 \leq i \leq M, 0 \leq k \leq N .
$$

Subtracting (31) from (26)-(28), (20c)-(20e), respectively, we obtain the error equations, as follows

$$
\begin{aligned}
\sum_{n=0}^{k} \hat{C}_{k-n}^{k+1}\left(\mathcal{A} \rho_{i}^{n+1}-\mathcal{A} \rho_{i}^{n}\right) & =\delta_{x}\left(\varkappa_{2} \delta_{x} e\right)_{i}^{k+\sigma}+\mathcal{A}\left(\tilde{F}_{i}^{k+\sigma}-\tilde{\mathcal{F}}_{i}^{k+\sigma}\right)+\mathcal{S}_{i}^{k+\sigma}, \quad 1 \leq i \leq M-1, \quad 0 \leq k \leq N-1, \\
\delta_{t}\left(\delta_{x}\left(\varkappa_{2} \delta_{x} e\right)_{i}^{1 / 2}\right) & =\delta_{x}\left(\varkappa_{2} \delta_{x} \rho\right)_{i}^{1 / 2}+s_{i}^{1 / 2}, \quad 1 \leq i \leq M-1, \\
\partial_{\hat{t}}\left(\delta_{x}\left(\varkappa_{2} \delta_{x} e\right)_{i}^{k+\sigma}\right) & =\delta_{x}\left(\varkappa_{2} \delta_{x} \rho\right)_{i}^{k+\sigma}+s_{i}^{k+\sigma}, \quad 1 \leq i \leq M-1, \quad 1 \leq k \leq N-1, \\
e_{i}^{k} & =0, \quad 1 \leq i \leq M-1, \quad-n_{0} \leq k \leq 0, \quad \rho_{i}^{0}=0, \quad 1 \leq i \leq M-1, \\
e_{0}^{k} & =e_{M}^{k}=0, \quad 0 \leq k \leq N \\
\rho_{0}^{k} & =\rho_{M}^{k}=0, \quad 0 \leq k \leq N .
\end{aligned}
$$

Theorem 1. Assume that $u(x, t) \in C_{x, t}^{6,4}([0, L] \times[-\tau, T])$ is the smooth solution of (5) and $\left\{\mathcal{V}_{i}^{k}, v_{i}^{k} \mid 0 \leq i \leq\right.$ $\left.M,-n_{0} \leq k \leq N\right\}$ the numerical solution of the scheme (31). Subsequently, there exist positive constants $h_{0}$ and $\tau_{0}$, independent of $h$ and $\tau$, such that, when $h \leq h_{0}$ and $\tau \leq \tau_{0}$, we have the error estimate

$$
\left\|e^{k}\right\|_{\infty} \leq C_{1}\left(\tau^{2}+h^{4}\right), \quad \tau \sum_{n=1}^{k}\left\|\rho^{n}\right\| \leq C_{1}\left(\tau^{2}+h^{4}\right), \quad-n_{0} \leq k \leq N .
$$

Proof. The proof will be preformed in two steps. Let us tackle the first one.

Step 1. When $k=0$, the system (51) is as follows

$$
\begin{aligned}
\hat{C}_{0}^{1}\left(\mathcal{A} \rho_{i}^{1}-\mathcal{A} \rho_{i}^{0}\right) & =\sigma \delta_{x}\left(\varkappa_{2} \delta_{x} e\right)_{i}^{1}+(1-\sigma) \delta_{x}\left(\varkappa_{2} \delta_{x} e\right)_{i}^{0}+\mathcal{S}_{i}^{\sigma}, \quad 1 \leq i \leq M-1, \\
\delta_{t}\left(\delta_{x}\left(\varkappa_{2} \delta_{x} e\right)_{i}^{1 / 2}\right) & =\delta_{x}\left(\varkappa_{2} \delta_{x} \rho\right)_{i}^{1 / 2}+s_{i}^{1 / 2}, \quad 1 \leq i \leq M-1, \\
e_{i}^{k} & =0, \quad 1 \leq i \leq M-1, \quad-n_{0} \leq k \leq 0, \quad \rho_{i}^{0}=0, \quad 1 \leq i \leq M-1, \\
e_{0}^{0} & =e_{M}^{0}=0 \\
\rho_{0}^{0} & =\rho_{M}^{0}=0 .
\end{aligned}
$$

Taking the inner product of (52a) with $\rho^{1}$, we obtain

$$
\begin{aligned}
\hat{C}_{0}^{1}\left\|\rho^{1}\right\|_{\mathcal{A}}^{2} & =\hat{C}_{0}^{1}\left\langle\mathcal{A} \rho^{0}, \rho^{1}\right\rangle+\left\langle\delta_{x}\left(\varkappa_{2} \delta_{x} e\right)^{\sigma}, \rho^{1}\right\rangle+\left\langle\mathcal{S}^{\sigma}, \rho^{1}\right\rangle \\
& =\hat{C}_{0}^{1}\left\langle\mathcal{A} \rho^{0}, \rho^{1}\right\rangle+\left\langle\sigma \delta_{x}\left(\varkappa_{2} \delta_{x} e\right)^{1}+(1-\sigma) \delta_{x}\left(\varkappa_{2} \delta_{x} e\right)^{0}, \rho^{1}\right\rangle+\left\langle\mathcal{S}^{\sigma}, \rho^{1}\right\rangle \\
& =\hat{C}_{0}^{1}\left\langle\mathcal{A} \rho^{0}, \rho^{1}\right\rangle-\sigma\left\langle e^{1}, \rho^{1}\right\rangle_{\varkappa_{2}}+(1-\sigma)\left\langle\delta_{x}\left(\varkappa_{2} \delta_{x} e\right)^{0}, \rho^{1}\right\rangle+\left\langle\mathcal{S}^{\sigma}, \rho^{1}\right\rangle,
\end{aligned}
$$

Taking the inner product of (52b) with $-2 \sigma e^{1}$ and invoking Lemma 3.3, we arrive at

$$
\frac{2 \sigma}{\tau}\left|e^{1}\right|_{1, \varkappa_{2}}^{2}=\frac{2 \sigma}{\tau}\left\langle e^{0}, e^{1}\right\rangle_{\varkappa_{2}}+\sigma\left(\left\langle e^{1}, \rho^{1}\right\rangle_{\varkappa_{2}}+\left\langle\rho^{0}, e^{1}\right\rangle_{\varkappa_{2}}\right)-2 \sigma\left\langle s^{1 / 2}, e^{1}\right\rangle .
$$

Adding (53) with (54) and using Young inequality and Lemma 2.6, we obtain 


$$
\begin{aligned}
\frac{2}{3} \hat{C}_{0}^{1}\left\|\rho^{1}\right\|^{2}+\frac{2 \sigma}{\tau}\left|e^{1}\right|_{1, \varkappa_{2}}^{2} \leq & \hat{C}_{0}^{1}\left\langle\mathcal{A} \rho^{0}, \rho^{1}\right\rangle+(1-\sigma)\left\langle\delta_{x}\left(\varkappa_{2} \delta_{x} e\right)^{0}, \rho^{1}\right\rangle+\left\langle\mathcal{S}^{\sigma}, \rho^{1}\right\rangle \\
& +\frac{2 \sigma}{\tau}\left\langle e^{0}, e^{1}\right\rangle_{\varkappa_{2}}+\sigma\left\langle\rho^{0}, e^{1}\right\rangle_{\varkappa_{2}}-2 \sigma\left\langle s^{1 / 2}, e^{1}\right\rangle \\
\leq & {\left[\frac{2}{9} \hat{C}_{0}^{1}\left\|\rho^{1}\right\|^{2}+\frac{9}{8} \hat{C}_{0}^{1}\left\|\rho^{0}\right\|^{2}\right]+\left[\frac{2 \hat{C}_{0}^{1}}{9}\left\|\rho^{1}\right\|^{2}+\frac{9(1-\sigma)}{8 \hat{C}_{0}^{1}}\left\|\delta_{x}\left(\varkappa_{2} \delta_{x} e\right)^{0}\right\|^{2}\right] } \\
& +\left[\frac{2 \hat{C}_{0}^{1}}{9}\left\|\rho^{1}\right\|^{2}+\frac{9}{8 \hat{C}_{0}^{1}}\left\|\mathcal{S}^{\sigma}\right\|^{2}\right]+\left[\frac{2 \sigma}{9 \tau}\left|e^{1}\right|_{1, \varkappa_{2}}^{2}+\frac{9 \sigma}{2 \tau}\left|e^{0}\right|_{1, \varkappa_{2}}^{2}\right] \\
& +\left[\frac{2 \sigma}{9 \tau}\left|e^{1}\right|_{1, \varkappa_{2}}^{2}+\frac{9 \tau \sigma}{8}\left|e^{0}\right|_{1, \varkappa_{2}}^{2}\right]+\left[\frac{2 \sigma}{9 \tau}\left|e^{1}\right|_{1, \varkappa_{2}}^{2}+\frac{3 \sigma \tau}{2} L^{2}\left\|s^{1 / 2}\right\|^{2}\right],
\end{aligned}
$$

after a simplification, we get

$$
\begin{aligned}
\left|e^{1}\right|_{1, \varkappa_{2}}^{2} \leq & \frac{27 \tau}{32 \sigma} \hat{C}_{0}^{1}\left\|\rho^{0}\right\|^{2}+\frac{27 \tau(1-\sigma)}{32 \sigma \hat{C}_{0}^{1}}\left\|\delta_{x}\left(\varkappa_{2} \delta_{x} e\right)^{0}\right\|^{2}+\frac{27 \tau}{32 \sigma \hat{C}_{0}^{1}}\left\|\mathcal{S}^{\sigma}\right\|^{2}+\frac{27}{8}\left|e^{0}\right|_{1, \varkappa_{2}}^{2} \\
& +\frac{27 \tau^{2}}{32}\left|e^{0}\right|_{1, \varkappa_{2}}^{2}+\frac{9 \tau^{2}}{8} L^{2}\left\|s^{1 / 2}\right\|^{2}
\end{aligned}
$$

it follows from $(52 b)$ that

$$
\delta_{x}\left(\varkappa_{2} \delta_{x} e\right)_{i}^{1}=\delta_{x}\left(\varkappa_{2} \delta_{x} e\right)_{i}^{0}+\tau \delta_{x}\left(\varkappa_{2} \delta_{x} \rho\right)_{i}^{1 / 2}+\tau s_{i}^{1 / 2}, \quad 1 \leq i \leq M-1 .
$$

Substituting (58) into (52a), we have

$$
\hat{C}_{0}^{1}\left(\mathcal{A} \rho_{i}^{1}-\mathcal{A} \rho_{i}^{0}\right)=\left[\tau \sigma \delta_{x}\left(\varkappa_{2} \delta_{x} \rho\right)_{i}^{1 / 2}+\tau s_{i}^{1 / 2}\right]+\delta_{x}\left(\varkappa_{2} \delta_{x} e\right)_{i}^{0}+\mathcal{S}_{i}^{\sigma}, \quad 1 \leq i \leq M-1 .
$$

Taking the inner product of (59) with $\rho^{1 / 2}$, we obtain

$$
\begin{aligned}
\hat{C}_{0}^{1}\left\langle\left(\mathcal{A} \rho^{1}-\mathcal{A} \rho^{0}\right), \rho^{1 / 2}\right\rangle=- & \tau \sigma\left|\rho^{1 / 2}\right|_{1, \varkappa_{2}}^{2}+\left\langle\tau \sigma \mathcal{S}^{1 / 2}, \rho^{1 / 2}\right\rangle+\left\langle\delta_{x}\left(\varkappa_{2} \delta_{x} e\right)^{0}, \rho^{1 / 2}\right\rangle \\
& +\left\langle\mathcal{S}^{\sigma}, \rho^{1 / 2}\right\rangle,
\end{aligned}
$$

By summation by parts and the Young inequality $a b \leq \frac{1}{2 \theta} a^{2}+\frac{\theta}{2} b^{2}$, with $\theta=\frac{3}{\hat{C}_{0}^{1}}$, this yields

$$
\begin{aligned}
\frac{\hat{C}_{0}^{1}}{2}\left(\left\|\rho^{1}\right\|_{\mathcal{A}}^{2}-\left\|\rho^{0}\right\|_{\mathcal{A}}^{2}\right)= & \left\langle\delta_{x}\left(\varkappa_{2} \delta_{x} e\right)^{0}, \rho^{1 / 2}\right\rangle-\tau \sigma\left|\rho^{1 / 2}\right|_{1, \varkappa_{2}}^{2}+\left\langle\mathcal{S}^{\sigma}, \rho^{1 / 2}\right\rangle+\left\langle\tau \sigma s^{1 / 2}, \rho^{1 / 2}\right\rangle \\
\leq & {\left[\frac{3}{2 \hat{C}_{0}^{1}}\left\|\delta_{x}\left(\varkappa_{2} \delta_{x} e\right)^{0}\right\|^{2}+\frac{\hat{C}_{0}^{1}}{6}\left\|\rho^{1 / 2}\right\|^{2}\right]+\left[\frac{3}{2 \hat{C}_{0}^{1}}\left\|\mathcal{S}^{\sigma}\right\|^{2}+\frac{\hat{C}_{0}^{1}}{6}\left\|\rho^{1 / 2}\right\|^{2}\right] } \\
& +\left[\frac{3 \tau^{2} \sigma^{2}}{2 \hat{C}_{0}^{1}}\left\|s^{1 / 2}\right\|^{2}+\frac{\hat{C}_{0}^{1}}{6}\left\|\rho^{1 / 2}\right\|^{2}\right] \\
\leq & \frac{\hat{C}_{0}^{1}}{4}\left(\left\|\rho^{0}\right\|^{2}+\left\|\rho^{1}\right\|^{2}\right)+\frac{3}{2 \hat{C}_{0}^{1}}\left\|\delta_{x}\left(\varkappa_{2} \delta_{x} e\right)^{0}\right\|^{2}+\frac{3}{2 \hat{C}_{0}^{1}}\left\|\mathcal{S}^{\sigma}\right\|^{2}+\frac{3 \tau^{2} \sigma^{2}}{2 \hat{C}_{0}^{1}}\left\|s^{1 / 2}\right\|^{2} .
\end{aligned}
$$

Subsequently, by invoking Lemma 2.6 and some simple manipulations, we get

$$
\left\|\rho^{1}\right\|^{2} \leq 9\left\|\rho^{0}\right\|^{2}+\frac{18}{\left(\hat{C}_{0}^{1}\right)^{2}}\left\|\delta_{x}\left(\varkappa_{2} \delta_{x} e\right)^{0}\right\|^{2}+\frac{18}{\left(\hat{C}_{0}^{1}\right)^{2}}\left\|\mathcal{S}^{\sigma}\right\|^{2}+\frac{18 \tau^{2} \sigma^{2}}{\left(\hat{C}_{0}^{1}\right)^{2}}\left\|s^{1 / 2}\right\|^{2}
$$

Step 2. When $k \geq 1$, we take the inner product of (51a) with $\rho^{k+\sigma}$ and obtain 


$$
\begin{aligned}
\left\langle\sum_{n=0}^{k} \hat{C}_{k-n}^{k+1}\left(\mathcal{A} \rho^{n+1}-\mathcal{A} \rho^{n}\right), \rho^{k+\sigma}\right\rangle & =\left\langle\delta_{x}\left(\varkappa_{2} \delta_{x} e\right)^{k+\sigma}, \rho^{k+\sigma}\right\rangle \\
+ & \left\langle\mathcal{A}\left(\tilde{F}^{k+\sigma}-\tilde{\mathcal{F}}^{k+\sigma}\right), \rho^{k+\sigma}\right\rangle+\left\langle\mathcal{S}^{k+\sigma}, \rho^{k+\sigma}\right\rangle, \quad 1 \leq k \leq N-1 .
\end{aligned}
$$

By Lemmas 2.4 and 2.10, we deduce for $1 \leq k \leq N-1$,

$$
\begin{aligned}
\left\langle\sum _ { n = 0 } ^ { k } \hat { C } _ { k - n } ^ { k + 1 } \left(\mathcal{A} \rho^{n+1}-\right.\right. & \left.\left.\mathcal{A} \rho^{n}\right), \rho^{k+\sigma}\right\rangle \geq \frac{1}{2} \sum_{n=0}^{k} \hat{C}_{k-n}^{k+1}\left[\left\|\rho^{n+1}\right\|_{\mathcal{A}}^{2}-\left\|\rho^{n}\right\|_{\mathcal{A}}^{2}\right] \\
& =\frac{1}{2}\left(\sum_{n=1}^{k+1} \hat{C}_{k-n+1}^{k+1}\left\|\rho^{n}\right\|_{\mathcal{A}}^{2}-\sum_{n=1}^{k} \hat{C}_{k-n}^{k}\left\|\rho^{n}\right\|_{\mathcal{A}}^{2}-\hat{b}_{k}\left\|\rho^{1}\right\|_{\mathcal{A}}^{2}-\hat{C}_{k}^{k+1}\left\|\rho^{0}\right\|_{\mathcal{A}}^{2}\right) .
\end{aligned}
$$

Young inequality is used for any $\theta>0$ to yield

$$
\left|\left\langle\mathcal{S}^{k+\sigma}, \rho^{k+\sigma}\right\rangle\right| \leq \theta\left\|\rho^{k+\sigma}\right\|^{2}+\frac{1}{4 \theta}\left\|\mathcal{S}^{k+\sigma}\right\|^{2}
$$

Using Lemma 3.1 and also Young inequality, this gives

$$
\begin{aligned}
\left\langle\mathcal{A}\left(\tilde{F}^{k+\sigma}-\tilde{\mathcal{F}}^{k+\sigma}\right), \rho^{k+\sigma}\right\rangle & \leq \theta\left\|\rho^{k+\sigma}\right\|^{2}+\frac{1}{4 \theta}\left\|\tilde{F}^{k+\sigma}-\tilde{\mathcal{F}}^{k+\sigma}\right\|_{\mathcal{A}}^{2} \\
& \leq \frac{\theta}{2}\left\|\rho^{k+\sigma}\right\|^{2}+\frac{1}{2 \theta}\left[2 c_{1}^{2}\left(\left\|\rho^{k}\right\|^{2}+\left\|\rho^{k-1}\right\|^{2}\right)+c_{2}^{2}\left(\left\|\rho^{k+1-n_{0}}\right\|^{2}+\left\|\rho^{k-n_{0}}\right\|^{2}\right)\right] .
\end{aligned}
$$

Substituting (65)-(67) in (64), we get, for all $1 \leq k \leq N-1$,

$$
\begin{aligned}
& \frac{1}{2}\left(\sum_{n=1}^{k+1} \hat{C}_{k-n+1}^{k+1}\left\|\rho^{n}\right\|_{\mathcal{A}}^{2}-\right.\left.\sum_{n=1}^{k} \hat{C}_{k-n}^{k}\left\|\rho^{n}\right\|_{\mathcal{A}}^{2}-\hat{b}_{k}\left\|\rho^{1}\right\|_{\mathcal{A}}^{2}-\hat{C}_{k}^{k+1}\left\|\rho^{0}\right\|_{\mathcal{A}}^{2}\right) \\
& \leq\left\langle\delta_{x}\left(\varkappa_{2} \delta_{x} e\right)^{k+\sigma}, \rho^{k+\sigma}\right\rangle+\theta\left\|\rho^{k+\sigma}\right\|^{2}+\frac{1}{4 \theta}\left\|\mathcal{S}^{k+\sigma}\right\|^{2} \\
&+\frac{\theta}{2}\left\|\rho^{k+\sigma}\right\|^{2}+\frac{1}{2 \theta}\left[2 c_{1}^{2}\left(\left\|\rho^{k}\right\|^{2}+\left\|\rho^{k-1}\right\|^{2}\right)+c_{2}^{2}\left(\left\|\rho^{k+1-n_{0}}\right\|^{2}+\left\|\rho^{k-n_{0}}\right\|^{2}\right)\right] .
\end{aligned}
$$

Taking the inner product of (51c) with $-e^{k+\sigma}$, we obtain

$$
-\left\langle\partial_{\hat{t}}\left(\delta_{x}\left(\varkappa_{2} \delta_{x} e\right)^{k+\sigma}\right), e^{k+\sigma}\right\rangle=-\left\langle\delta_{x}\left(\varkappa_{2} \delta_{x} \rho\right)^{k+\sigma}, e^{k+\sigma}\right\rangle-\left\langle s^{k+\sigma}, e^{k+\sigma}\right\rangle, \quad 1 \leq k \leq N-1 .
$$

For the 1.h.s of (69), after recalling Lemmas 2.10 and 3.3, we get

$$
-\left\langle\partial_{\hat{t}}\left(\delta_{x}\left(\varkappa_{2} \delta_{x} e\right)^{k+\sigma}\right), e^{k+\sigma}\right\rangle \geq \frac{1}{4 \tau}\left(\tilde{\mathcal{E}}^{k+1}-\tilde{\mathcal{E}}^{k}\right), \quad 1 \leq k \leq N-1,
$$

such that

$$
\tilde{\mathcal{E}}^{k+1}=(2 \sigma+1)\left|e^{k+1}\right|_{1, \varkappa_{2}}^{2}-(2 \sigma-1)\left|e^{k}\right|_{1, \varkappa_{2}}^{2}+\left(2 \sigma^{2}+\sigma-1\right)\left|e^{k+1}-e^{k}\right|_{1, \varkappa_{2}}^{2} \quad k \geq 0,
$$

and additionally,

$$
\tilde{\mathcal{E}}^{k+1} \geq \frac{1}{\sigma}\left|e^{k+1}\right|_{1, \varkappa_{2}}^{2} \quad k \geq 0
$$


By the Cauchy-Schwarz inequality, we have

$$
\left|-\left\langle s^{k+\sigma}, e^{k+\sigma}\right\rangle\right| \leq \frac{1}{2}\left\|e^{k+\sigma}\right\|^{2}+\frac{1}{2}\left\|s^{k+\sigma}\right\|^{2}, \quad 1 \leq k \leq N-1,
$$

so plugging (70) and (72) into (69), this gives

$$
\frac{1}{4 \tau}\left(\tilde{\mathcal{E}}^{k+1}-\tilde{\mathcal{E}}^{k}\right) \leq-\left\langle\delta_{x}\left(\varkappa_{2} \delta_{x} \rho\right)^{k+\sigma}, e^{k+\sigma}\right\rangle+\frac{1}{2}\left\|e^{k+\sigma}\right\|^{2}+\frac{1}{2}\left\|s^{k+\sigma}\right\|^{2}, \quad 1 \leq k \leq N-1 .
$$

Adding (68) and (73), we obtain

$$
\begin{aligned}
\frac{1}{2}\left(\sum_{n=1}^{k+1} \hat{C}_{k-n+1}^{k+1}\left\|\rho^{n}\right\|_{\mathcal{A}}^{2}-\right. & \left.\sum_{n=1}^{k} \hat{C}_{k-n}^{k}\left\|\rho^{n}\right\|_{\mathcal{A}}^{2}-\hat{b}_{k}\left\|\rho^{1}\right\|_{\mathcal{A}}^{2}-\hat{C}_{k}^{k+1}\left\|\rho^{0}\right\|_{\mathcal{A}}^{2}\right)+\frac{1}{4 \tau}\left(\tilde{\mathcal{E}}^{k+1}-\tilde{\mathcal{E}}^{k}\right) \\
\leq & \frac{1}{2}\left\|\rho^{k+\sigma}\right\|^{2}+\frac{1}{2}\left\|s^{k+\sigma}\right\|^{2}+\theta\left\|\rho^{k+\sigma}\right\|^{2}+\frac{1}{4 \theta}\left\|\mathcal{S}^{k+\sigma}\right\|^{2} \\
& \quad+\frac{\theta}{2}\left\|\rho^{k+\sigma}\right\|^{2}+\frac{1}{2 \theta}\left[2 c_{1}^{2}\left(\left\|\rho^{k}\right\|^{2}+\left\|\rho^{k-1}\right\|^{2}\right)+c_{2}^{2}\left(\left\|\rho^{k+1-n_{0}}\right\|^{2}+\left\|\rho^{k-n_{0}}\right\|^{2}\right)\right] .
\end{aligned}
$$

Now, multiply both sides of (74) by $4 \tau$, use Lemma 2.6 and do some arrangements, we then have

$$
\begin{aligned}
2 \tau \sum_{n=1}^{k+1} \hat{C}_{k-n+1}^{k+1}\left\|\rho^{n}\right\|_{\mathcal{A}}^{2}+\tilde{\mathcal{E}}^{k+1} \leq & \left.2 \tau\left(\sum_{n=1}^{k} \hat{C}_{k-n}^{k}\left\|\rho^{n}\right\|_{\mathcal{A}}^{2}\right)\right)+\tilde{\mathcal{E}}^{k}+2 \tau\left(\hat{b}_{k}\left\|\rho^{1}\right\|^{2}+\hat{C}_{k}^{k+1}\left\|\rho^{0}\right\|^{2}\right) \\
& +\frac{4 \tau}{2}\left\|e^{k+\sigma}\right\|^{2}+\frac{4 \tau}{2}\left\|s^{k+\sigma}\right\|^{2}+\frac{4 \tau}{4 \theta}\left\|\mathcal{S}^{k+\sigma}\right\|^{2}+2 \tau(1+2 \theta)\left\|\rho^{k+\sigma}\right\|^{2} \\
& +\frac{4 \tau}{2}\left[2 c_{1}^{2}\left(\left\|\rho^{k}\right\|^{2}+\left\|\rho^{k-1}\right\|^{2}\right)+c_{2}^{2}\left(\left\|\rho^{k+1-n_{0}}\right\|^{2}+\left\|\rho^{k-n_{0}}\right\|^{2}\right)\right] .
\end{aligned}
$$

Denote $\mathcal{H}^{k+1}=2 \tau \sum_{n=1}^{k+1} \hat{C}_{k-n+1}^{k+1}\left\|\rho^{n}\right\|_{\mathcal{A}}^{2}+\tilde{\mathcal{E}}^{k+1}$, we can write

$$
\begin{aligned}
\mathcal{H}^{k+1} \leq & \mathcal{H}^{k}+2 \tau\left(\hat{b}_{k}\left\|\rho^{1}\right\|^{2}+\hat{C}_{k}^{k+1}\left\|\rho^{0}\right\|^{2}\right)+\frac{4 \tau}{2}\left\|e^{k+\sigma}\right\|^{2}+\frac{4 \tau}{2}\left\|s^{k+\sigma}\right\|^{2}+\frac{4 \tau}{4 \theta}\left\|\mathcal{S}^{k+\sigma}\right\|^{2} \\
& +8 \tau \theta\left\|\rho^{k+\sigma}\right\|^{2}+\frac{4 \tau}{\theta}\left[2 c_{1}^{2}\left(\left\|\rho^{k}\right\|^{2}+\left\|\rho^{k-1}\right\|^{2}\right)+c_{2}^{2}\left(\left\|\rho^{k+1-n_{0}}\right\|^{2}+\left\|\rho^{k-n_{0}}\right\|^{2}\right)\right] \\
\leq & \mathcal{H}^{1}+2 \tau\left(\sum_{n=1}^{k} \hat{b}_{n}\left\|\rho^{1}\right\|^{2}+\sum_{n=1}^{k} \hat{C}_{n}^{k+1}\left\|\rho^{0}\right\|^{2}\right)+\frac{8 \tau}{2} \sum_{n=1}^{k+1}\left\|e^{n}\right\|^{2}+\frac{4 \tau}{2} \sum_{n=1}^{k}\left\|s^{n+\sigma}\right\|^{2} \\
& +\frac{4 \tau}{4 \theta} \sum_{n=1}^{k}\left\|\mathcal{S}^{n+\sigma}\right\|^{2}+4 \tau(1+2 \theta) \sum_{n=1}^{k+1}\left\|\rho^{n}\right\|^{2}+\frac{4 \tau}{2} \sum_{n=1}^{k}\left[2 c_{1}^{2}\left(\left\|\rho^{n}\right\|^{2}+\left\|\rho^{n-1}\right\|^{2}\right)\right. \\
& \left.+c_{2}^{2}\left(\left\|\rho^{n+1-n_{0}}\right\|^{2}+\left\|\rho^{n-n_{0}}\right\|^{2}\right)\right], \quad 1 \leq k \leq N-1 .
\end{aligned}
$$

From (71) and Lemma 2.4,

$$
\begin{gathered}
\mathcal{H}^{k+1} \geq \tau \sum_{r=0}^{m} p_{r} \frac{\left(1-\gamma_{r}\right) T^{-\gamma_{r}}}{\Gamma\left(2-\gamma_{r}\right)}(k+\sigma)^{1-\gamma_{r}} \sum_{n=1}^{k+1}\left\|\rho^{n}\right\|_{\mathcal{A}}^{2}+\frac{1}{\sigma}\left|e^{k+1}\right|_{1, \varkappa_{2}{ }^{\prime}}^{2} \quad 1 \leq k \leq N-1, \\
\mathcal{H}^{1}=2 \tau \hat{C}_{0}^{1}\left\|\rho^{1}\right\|_{\mathcal{A}}^{2}+\mathcal{E}^{1} \leq 2 \tau \hat{C}_{0}^{1}\left\|\rho^{1}\right\|^{2}+(2 \sigma+1)\left|e^{1}\right|_{1, \varkappa_{2}}^{2}+\left(2 \sigma^{2}+\sigma-1\right)\left|e^{1}\right|_{1, \varkappa_{2}}^{2} .
\end{gathered}
$$


Inserting the above two inequalities into (76) and considering

$$
c:=\max \left\{c_{1}, c_{2}\right\}, \quad \mathrm{A}:=\frac{2}{3} \sum_{r=0}^{m} p_{r} \frac{\left(1-\gamma_{r}\right) T^{-\gamma_{r}}}{\Gamma\left(2-\gamma_{r}\right)}(k+\sigma)^{1-\gamma_{r}},
$$

yields

$$
\begin{aligned}
\frac{1}{\sigma}\left|e^{k+1}\right|_{1, \varkappa_{2}}^{2}+\tau & A \sum_{n=1}^{k+1}\left\|\rho^{n}\right\|^{2} \leq 2 \tau \hat{C}_{0}^{1}\left\|\rho^{1}\right\|^{2}+\left(2 \sigma^{2}+3 \sigma\right)\left|e^{1}\right|_{1, \varkappa_{2}}^{2} \\
+ & 2 \tau\left(\sum_{n=1}^{k} \hat{b}_{n}\left\|\rho^{1}\right\|^{2}+\sum_{n=1}^{k} \hat{C}_{n}^{k+1}\left\|\rho^{0}\right\|^{2}\right)+4 \tau \sum_{n=1}^{k+1}\left\|e^{n}\right\|^{2}+2 \tau \sum_{n=1}^{k}\left\|s^{n+\sigma}\right\|^{2} \\
& +\frac{\tau}{\theta} \sum_{n=1}^{k}\left\|\mathcal{S}^{n+\sigma}\right\|^{2}+4 \tau\left(1+2 \theta+3 c^{2}\right) \sum_{n=1}^{k+1}\left\|\rho^{n}\right\|^{2} .
\end{aligned}
$$

By choosing $\theta$ to achieve $\mathrm{A} \geq 4\left(1+2 \theta+3 c^{2}\right)$, invoking (57), (63), and denoting

$$
\begin{aligned}
\mathcal{G}_{k+1}:= & 2 \tau\left(\hat{C}_{0}^{1}+\sum_{n=1}^{k} \hat{b}_{n}\right)\left[9\left\|\rho^{0}\right\|^{2}+\frac{18}{\left(\hat{C}_{0}^{1}\right)^{2}}\left\|\delta_{x}\left(\varkappa_{2} \delta_{x} e\right)^{0}\right\|^{2}+\frac{18}{\left(\hat{C}_{0}^{1}\right)^{2}}\left\|\mathcal{S}^{\sigma}\right\|^{2}+\frac{18 \tau^{2} \sigma^{2}}{\left(\hat{C}_{0}^{1}\right)^{2}}\left\|s^{1 / 2}\right\|^{2}\right] \\
& +\left(2 \sigma^{2}+3 \sigma\right)\left[\frac{27 \tau}{32 \sigma} \hat{C}_{0}^{1}\left\|\rho^{0}\right\|^{2}+\frac{27 \tau(1-\sigma)}{32 \sigma \hat{C}_{0}^{1}}\left\|\delta_{x}\left(\varkappa_{2} \delta_{x} e\right)^{0}\right\|^{2}+\frac{27 \tau}{32 \sigma \hat{C}_{0}^{1}}\left\|\mathcal{S}^{\sigma}\right\|^{2}+\frac{27}{8}\left|e^{0}\right|_{1, \varkappa_{2}}^{2}\right. \\
& \left.+\frac{27 \tau^{2}}{32}\left|e^{0}\right|_{1, \varkappa_{2}}^{2}+\frac{9 \tau^{2}}{8} L^{2}\left\|s^{1 / 2}\right\|^{2}\right]+2 \tau \sum_{n=1}^{k} \hat{C}_{n}^{k+1}\left\|\rho^{0}\right\|^{2}+2 \tau \sum_{n=1}^{k}\left\|s^{n+\sigma}\right\|^{2}+\frac{\tau}{\theta} \sum_{n=1}^{k}\left\|\mathcal{S}^{n+\sigma}\right\|^{2},
\end{aligned}
$$

we obtain directly after following the assumptions (13),

$$
\left|e^{k+1}\right|_{1}^{2} \leq \frac{1}{b_{2}}\left|e^{k+1}\right|_{1, \varkappa_{2}}^{2} \leq \frac{4 \tau \sigma}{b_{2}} \sum_{n=1}^{k+1}\left\|e^{n}\right\|^{2}+\frac{\sigma}{b_{2}} \mathcal{G}_{k+1}, \quad 1 \leq k \leq N-1,
$$

invoking Lemma 2.6 gives

$$
\left|e^{k+1}\right|_{1}^{2} \leq \frac{2 \tau L^{2} \sigma}{3 b_{2}} \sum_{n=1}^{k+1}\left|e^{n}\right|_{1}^{2}+\frac{\sigma}{b_{2}} \mathcal{G}_{k+1}, \quad 1 \leq k \leq N-1,
$$

applying Grönwall Lemma 3.2 yields

$$
\left|e^{k+1}\right|_{1}^{2} \leq \frac{\sigma}{b_{2}} \exp \left(\frac{4 L^{2} \sigma}{3 b_{2}}\right) \mathcal{G}_{k+1}, \quad 1 \leq k \leq N-1 .
$$

Accordingly, the proof is completed.

\section{Almost Unconditional Stability}

To discuss the stability of the compact difference scheme (31), we also use the discrete energy method. Let $\left\{\overline{\mathcal{V}}_{i}^{k}, \bar{v}_{i}^{k} \mid 0 \leq i \leq M,-n_{0} \leq k \leq N\right\}$ be the solution of 


$$
\begin{aligned}
\sum_{n=0}^{k} \hat{C}_{k-n}^{k+1}\left(\mathcal{A} \overline{\mathcal{V}}_{i}^{n+1}-\mathcal{A} \overline{\mathcal{V}}_{i}^{n}\right) & =\delta_{x}\left(\varkappa_{2} \delta_{x} \bar{v}\right)_{i}^{k+\sigma}+\mathcal{A} \tilde{\mathbb{F}}_{i}^{k+\sigma}, \quad 1 \leq i \leq M-1, \quad 0 \leq k \leq N-1, \\
\delta_{t}\left(\delta_{x}\left(\varkappa_{2} \delta_{x} \bar{v}\right)_{i}^{1 / 2}\right) & =\delta_{x}\left(\varkappa_{2} \delta_{x} \bar{v}\right)_{i}^{1 / 2}, \quad 1 \leq i \leq M-1, \\
\partial_{\hat{t}}\left(\delta_{x}\left(\varkappa_{2} \delta_{x} \bar{v}\right)_{i}^{k+\sigma}\right) & =\delta_{x}\left(\varkappa_{2} \delta_{x} \bar{v}\right)_{i}^{k+\sigma}, \quad 1 \leq i \leq M-1, \quad 1 \leq k \leq N-1, \\
\bar{v}_{i}^{k} & =\hat{v}_{i}^{k}+e_{i}^{k}, \quad 1 \leq i \leq M-1, \quad-n_{0} \leq k \leq 0, \quad \bar{v}_{i}^{0}=\hat{\psi}\left(x_{i}\right), \quad 1 \leq i \leq M-1, \\
\bar{v}_{0}^{k} & =\bar{v}_{M}^{k}=0, \quad 0 \leq k \leq N, \\
\overline{\mathcal{V}}_{0}^{k} & =\overline{\mathcal{V}}_{M}^{k}=0, \quad 0 \leq k \leq N
\end{aligned}
$$

where

$$
\tilde{\mathbb{F}}_{i}^{k+\sigma}=\tilde{f}\left((\sigma+1) \bar{v}_{i}^{k}-\sigma \bar{v}_{i}^{k-1}, \sigma \bar{v}_{i}^{k+1-n_{0}}+(1-\sigma) \bar{v}_{i}^{k-n_{0}}, x_{i}, t_{k+\sigma}\right) .
$$

where $\varrho_{i}^{k}$ denotes an initial perturbation term that is very small.

Theorem 2. Let $\bar{\rho}_{i}^{k}=\overline{\mathcal{V}}_{i}^{k}-\mathcal{V}_{i}^{k}, \quad \bar{e}_{i}^{k}=\bar{v}_{i}^{k}-v_{i}^{k}$, for $0 \leq i \leq M,-n_{0} \leq k \leq N$. Subsequently, there exist constants $c_{4}, c_{5}, h_{0}, \tau_{0}$ that fulfill

$$
\left\|\bar{e}^{k}\right\|_{\infty} \leq c_{4} \sum_{k=-n}^{0}\left\|\varrho^{k}\right\|, \quad 0 \leq k \leq N
$$

conditioned by

$$
h \leq h_{0}, \quad \tau \leq \tau_{0}, \quad \max _{\substack{-n \leq k \leq 0 \\ 0 \leq i \leq M}}\left|\bar{e}_{i}^{k}\right| \leq c_{5} .
$$

Proof. The perturbation equations in terms of $\bar{\rho}_{i}^{k}$ and $\bar{e}_{i}^{k}$ come by subtracting (82) from (31) and similar to the proof of Theorem 1, the conclusion of stability holds immediately.

\section{Generalized Scheme for the Distributed Order Case}

We are now in a position to consider the distributed order form of dmfCDWEs, which means that

$$
\int_{1}^{2} \omega(\alpha) \frac{\partial^{\alpha} u(x, t)}{\partial t^{\alpha}} d \alpha=\frac{\partial}{\partial x}\left(q_{1}(x) \frac{\partial u}{\partial x}\right)+q_{2}(x) \frac{\partial u}{\partial x}+f(u(x, t), u(x, t-s), x, t), 0<t \leq T, 0 \leq x \leq L,
$$

with the following initial and boundary conditions

$$
\begin{aligned}
& u(x, t)=d(x, t), \quad 0 \leq x \leq L, \quad t \in[-s, 0), \quad \frac{\partial u(x, 0)}{\partial t}=\psi(x)=\lim _{t \rightarrow-0} \frac{\partial d(x, t)}{\partial t} \\
& u(0, t)=\phi_{0}(t), \quad u(L, t)=\phi_{L}(t), \quad 0<t \leq T
\end{aligned}
$$

Following the same manipulations illustrated before; starting from an exponential transformation technique, then a transformation to zero Dirichlet boundary conditions, we obtain the following system

$$
\int_{1}^{2} \omega(\alpha) \frac{\partial^{\alpha} v(x, t)}{\partial t^{\alpha}} d \alpha=\frac{\partial}{\partial x}\left(q_{1}(x) \frac{\partial v}{\partial x}\right)+\tilde{f}(v(x, t), v(x, t-s), x, t), 0<t \leq T, 0 \leq x \leq L,
$$

with the initial and boundary conditions as

$$
\begin{aligned}
& v(x, t)=\hat{r}(x, t), \quad 0 \leq x \leq L, \quad t \in[-s, 0), \quad \frac{\partial v(x, 0)}{\partial t}=\hat{\psi}(x)=\lim _{t \rightarrow-0} \frac{\partial r(x, t)}{\partial t}, \\
& v(0, t)=v(L, t)=0, \quad t>0 .
\end{aligned}
$$


A numerical quadrature rule can be adapted to transform the system (85) to dmfCDWEs. We recall Simpson's rule (also known as the three-point Newton-Cotes quadrature rule), a proof of which can be found in any descent textbook.

Lemma 5.1. Consider an equidistant partition of the interval $[1,2]$ into $2 J$ subintervals, let $\Delta \alpha=\frac{1}{2 J}$ and denote $\alpha_{l}=1+l \Delta \alpha, 0 \leq l \leq 2 J$. Subsequently, the composite Simpson's rule reads

$$
\int_{1}^{2} f(\alpha) d \alpha=\Delta \alpha \sum_{l=0}^{2 J} \gamma_{l} f\left(\alpha_{l}\right)-\frac{(\Delta \alpha)^{4}}{180} f^{(4)}(\zeta), \quad \zeta \in[1,2]
$$

where

$$
\gamma_{l}= \begin{cases}\frac{1}{3}, & l=0,2 J \\ \frac{2}{3}, & l=2,4, \ldots, 2 J-4,2 J-2 \\ \frac{4}{3}, & l=1,3, \ldots, 2 J-3,2 J-1\end{cases}
$$

Define the function $G\left(\cdot ; x_{i}, t_{j}\right): \alpha \mapsto \omega(\alpha) \frac{\partial^{\alpha} v\left(x_{i}, t_{j}\right)}{\partial t^{\alpha}}$. Suppose that $G(\alpha) \in C^{4}([1,2])$, then by using Lemma 5.1, we approximate the distributed derivative as

$$
\begin{aligned}
\int_{1}^{2} \omega(\alpha) \frac{\partial^{\alpha} v\left(x_{i}, t_{k+\sigma}\right)}{\partial t^{\alpha}} d \alpha & =\Delta \alpha \sum_{l=0}^{2 J} \gamma_{l} \omega\left(\alpha_{l}\right){ }_{0}^{C} D_{t}^{\alpha_{l}} v\left(x_{i}, t_{k-1 / 2}\right)-\left.\frac{(\Delta \alpha)^{4}}{180} G^{(4)}\left(\alpha ; x_{i}, t_{k+\sigma}\right)\right|_{\alpha=\zeta_{i}^{k}}, \\
& =\Delta \alpha \sum_{l=0}^{2 J} \gamma_{l} \omega\left(\alpha_{l}\right){ }_{0}^{C} D_{t}^{\alpha_{l}} v\left(x_{i}, t_{k+\sigma}\right)+O(\Delta \alpha)^{4},
\end{aligned}
$$

for a $\zeta_{i}^{k} \in[1,2]$. Define

$$
\hat{\bar{C}}_{n}^{(k+1)}=\Delta \alpha \sum_{l=0}^{2 J} \gamma_{l} \omega\left(\alpha_{l}\right) \frac{\tau^{-\gamma_{l}}}{\Gamma\left(2-\gamma_{l}\right)} C_{n}^{\left(k+1, \gamma_{l}\right)}, \quad n=0,1, \ldots, k,
$$

then the constructed compact difference scheme has the following form

$$
\begin{aligned}
\sum_{n=0}^{k} \hat{\bar{C}}_{k-n}^{k+1}\left(\mathcal{A} \mathcal{V}_{i}^{n+1}-\mathcal{A} \mathcal{V}_{i}^{n}\right) & =\delta_{x}\left(\varkappa_{2} \delta_{x} v\right)_{i}^{k+\sigma}+\mathcal{A} \tilde{\mathcal{F}}_{i}^{k+\sigma}, \quad 1 \leq i \leq M-1, \quad 0 \leq k \leq N-1, \\
\delta_{t}\left(\delta_{x}\left(\varkappa_{2} \delta_{x} v\right)_{i}^{1 / 2}\right) & =\delta_{x}\left(\varkappa_{2} \delta_{x} \mathcal{V}\right)_{i}^{1 / 2}, \quad 1 \leq i \leq M-1, \\
\partial_{\hat{t}}\left(\delta_{x}\left(\varkappa_{2} \delta_{x} v\right)_{i}^{k+\sigma}\right) & =\delta_{x}\left(\varkappa_{2} \delta_{x} \mathcal{V}\right)_{i}^{k+\sigma}, \quad 1 \leq i \leq M-1, \quad 1 \leq k \leq N-1, \\
v_{i}^{k} & =\hat{r}_{i}^{k}, \quad 1 \leq i \leq M-1, \quad-n_{0} \leq k \leq 0, \quad \mathcal{V}_{i}^{0}=\hat{\psi}\left(x_{i}\right), \quad 1 \leq i \leq M-1, \\
v_{0}^{k} & =v_{M}^{k}=0, \quad 0 \leq k \leq N, \\
\mathcal{V}_{0}^{k} & =\mathcal{V}_{M}^{k}=0, \quad 0 \leq k \leq N .
\end{aligned}
$$

Remark 1. The local truncation error of the compact difference scheme (88) for the distributed order system (85) is of order $\mathcal{O}\left(\tau^{2}+h^{4}+(\Delta \alpha)^{4}\right)$. The convergence and stability estimates can be derived in the same manner as in Theorem 1 and Theorem 2.

\section{Numerical Illustration}

The purpose of the present section is to demonstrate the convergence rate of the method. We will consider the maximum absolute error between the exact solution $u\left(x_{i}, t_{k}\right)$ of the continuous problem and corresponding approximations $u_{i}^{k}$, which is given by

$$
\epsilon_{\tau, h}=\max _{0 \leq i \leq M, 0 \leq k \leq N}\left|u\left(x_{i}, t_{n}\right)-u_{i}^{n}\right|
$$


Moreover, we define the standard rates

$$
\rho_{\tau, h}^{x}=\log _{2}\left(\frac{\epsilon_{\tau, 2 h}}{\epsilon_{\tau, h}}\right), \& \quad \& \rho_{\tau, h}^{t}=\log _{2}\left(\frac{\epsilon_{2 \tau, h}}{\epsilon_{\tau, h}}\right) .
$$

We consider the following multiterm time fractional delay sup-diffusion problem

$$
\begin{gathered}
\sum_{r=0}^{2} p_{r} \frac{\partial^{\alpha_{r}} u(x, t)}{\partial t^{\alpha_{r}}}=\frac{(x+1)}{2} \frac{\partial^{2} u}{\partial x^{2}}+(x+1)^{2} \frac{\partial u}{\partial x}+f(u(x, t), u(x, t-0.2), x, t), \\
f(u(x, t), u(x, t-0.2), x, t)=-u^{2}(t, x)+u(t-0.2, x)+g(x, t), \quad \forall(x, t) \in[0,1] \times[0,1] .
\end{gathered}
$$

Note that $g(x, t)$ is defined/derived, such that $u(x, t)=e^{x} x^{2}(1-x)^{2} t^{3}$ is the exact solution. The exact solution determines the initial condition and boundary conditions. The difference scheme (31) is employed in order to obtain the numerical solution. First, the numerical accuracy of this scheme in time will be verified. Taking a sufficiently small step size $h$ and varying step size $\tau$, the numerical errors and numerical convergence orders are listed in the lower half of Table 1. The computational results presented in Table 1 confirm the second-order convergence of the difference scheme (31) in time.

Table 1. Absolute errors and standard convergence rates in space and time when approximating

\begin{tabular}{|c|c|c|c|c|c|}
\hline \multicolumn{6}{|c|}{ Spatial Analysis of Convergence } \\
\hline \multirow[b]{2}{*}{$\tau$} & \multirow[b]{2}{*}{$h$} & \multicolumn{2}{|c|}{$\left(p_{0}=1, p_{1}=1.25, p_{2}=2\right)$} & \multicolumn{2}{|c|}{$\left(p_{0}=2, p_{1}=1.75, p_{2}=1\right)$} \\
\hline & & $\epsilon_{\tau, h}$ & $\rho_{\tau, h}^{x}$ & $\epsilon_{\tau, h}$ & $\rho_{\tau, h}^{x}$ \\
\hline \multirow{4}{*}{0.001} & $0.02 \times 2^{-1}$ & $1.877 \times 10^{-3}$ & - & $4.179 \times 10^{-4}$ & - \\
\hline & $0.02 \times 2^{-2}$ & $1.388 \times 10^{-4}$ & 3.765 & $2.676 \times 10^{-5}$ & 3.965 \\
\hline & $0.02 \times 2^{-3}$ & $9.733 \times 10^{-6}$ & 3.834 & $1.697 \times 10^{-6}$ & 3.979 \\
\hline & $0.02 \times 2^{-4}$ & $6.306 \times 10^{-7}$ & 3.948 & $1.066 \times 10^{-7}$ & 3.992 \\
\hline \multirow{4}{*}{0.0005} & $0.02 \times 2^{-1}$ & $2.480 \times 10^{-4}$ & - & $6.981 \times 10^{-5}$ & - \\
\hline & $0.02 \times 2^{-2}$ & $1.601 \times 10^{-5}$ & 3.953 & $4.430 \times 10^{-6}$ & 3.978 \\
\hline & $0.02 \times 2^{-3}$ & $1.016 \times 10^{-6}$ & 3.978 & $2.791 \times 10^{-7}$ & 3.988 \\
\hline & $0.02 \times 2^{-4}$ & $6.399 \times 10^{-8}$ & 3.989 & $1.750 \times 10^{-8}$ & 3.995 \\
\hline \multicolumn{6}{|c|}{ Temporal Analysis of Convergence } \\
\hline \multirow[b]{2}{*}{$h$} & \multirow[b]{2}{*}{$\tau$} & \multicolumn{2}{|c|}{$\left(p_{0}=1, p_{1}=1.25, p_{2}=2\right)$} & \multicolumn{2}{|c|}{$\left(p_{0}=2, p_{1}=1.75, p_{2}=1\right)$} \\
\hline & & $\epsilon_{\tau, h}$ & $\rho_{\tau, h}^{t}$ & $\epsilon_{\tau, h}$ & $\rho_{\tau, h}^{t}$ \\
\hline \multirow{4}{*}{0.005} & $0.01 \times 2^{-1}$ & $3.357 \times 10^{-4}$ & - & $7.278 \times 10^{-5}$ & - \\
\hline & $0.01 \times 2^{-2}$ & $9.152 \times 10^{-5}$ & 1.875 & $1.842 \times 10^{-5}$ & 1.984 \\
\hline & $0.01 \times 2^{-3}$ & $2.395 \times 10^{-5}$ & 1.934 & $4.640 \times 10^{-6}$ & 1.989 \\
\hline & $0.01 \times 2^{-4}$ & $7.450 \times 10^{-6}$ & 1.978 & $1.164 \times 10^{-6}$ & 1.995 \\
\hline \multirow{4}{*}{0.001} & $0.01 \times 2^{-1}$ & $9.375 \times 10^{-5}$ & - & $5.378 \times 10^{-6}$ & - \\
\hline & $0.01 \times 2^{-2}$ & $2.472 \times 10^{-5}$ & 1.923 & $1.359 \times 10^{-6}$ & 1.987 \\
\hline & $0.01 \times 2^{-3}$ & $6.366 \times 10^{-6}$ & 1.964 & $3.409 \times 10^{-7}$ & 1.994 \\
\hline & $0.01 \times 2^{-4}$ & $1.592 \times 10^{-6}$ & 1.993 & $8.534 \times 10^{-8}$ & 1.998 \\
\hline
\end{tabular}
the solution $u$ of (1) with $\left(\alpha_{1}=1.3, \alpha_{2}=1.5, \alpha_{3}=1.7\right)$, while using the difference method (31). The parameters and conditions employed in this case correspond to those in Example 6.

Next, the numerical accuracy of the difference scheme in space for solving this example is examined. The numerical results of this scheme for different step sizes in space are calculated and the numerical errors, as well as the numerical convergence orders are recorded in the upper half of Table 1. Again, from which, one can find that, in this case the fourth-order convergence is achieved. 


\section{Conclusions}

A linearized difference scheme for solving a class of dmfCDWEs is constructed. With the help of an easy to execute and invertible exponential transformation, the considered problem can be converted into the delay variable coefficient fractional diffusion wave equation equivalently. Subsequently, we establish a fourth-order accurate numerical scheme that is based on a variable coefficient compact operator and with a temporal second order of convergence at a super-convergent point. The convergence and stability of the current numerical scheme are proved at length and a numerical example is finally added for the sake of demonstrating the theoretical findings.

Author Contributions: All authors contributed equally. All authors have read and agreed to the published version of the manuscript.

Funding: The first author wishes to acknowledge the support of RFBR Grant 19-01-00019.

Acknowledgments: The authors wish to thank the anonymous reviewers for their comments and criticism. All of their comments were taken into account in the revised version of the paper, resulting in a substantial improvement with respect to the original submission.

Conflicts of Interest: The authors declare no conflict of interest.

\section{References}

1. Metzler, R.; Klafter, J. The random walk's guide to anomalous diffusion: A fractional dynamics approach. Phys. Rep. 2000, 339, 1-77. [CrossRef]

2. Sun, Z.Z.; Wu, X. A fully discrete difference scheme for a diffusion-wave system. Appl. Numer. Math. 2006, 56, 193-209. [CrossRef]

3. Alikhanov, A. A new difference scheme for the fractional diffusion equation. J. Comput. Phys. 2015, 280, 424-438. [CrossRef]

4. Gafiychuk, V.; Datsko, B.Y. Pattern formation in a fractional reaction-diffusion system. Phys. A Stat. Mech. Appl. 2006, 365, 300-306. [CrossRef]

5. Tomovski, Ž.; Hilfer, R.; Srivastava, H. Fractional and operational calculus with generalized fractional derivative operators and Mittag-Leffler type functions. Integral Transform. Spec. Funct. 2010, 21, 797-814. [CrossRef]

6. Zhu, B.; Liu, L.; Wu, Y. Existence and uniqueness of global mild solutions for a class of nonlinear fractional reaction-diffusion equations with delay. Comput. Math. Appl. 2019, 78, 1811-1818. [CrossRef]

7. Hendy, A.S.; De Staelen, R.H.; Pimenov, V.G. A semi-linear delayed diffusion-wave system with distributed order in time. Numer. Algorithms 2018, 77, 885-903. [CrossRef]

8. Gao, G.H.; Alikhanov, A.A.; Sun, Z.Z. The temporal second order difference schemes based on the interpolation approximation for solving the time multi-term and distributed-order fractional sub-diffusion equations. J. Sci. Comput. 2017, 73, 93-121. [CrossRef]

9. Sun, Z.Z. An unconditionally stable and $O\left(\tau^{2}+h^{4}\right)$ order $L_{\infty}$ convergent difference scheme for linear parabolic equations with variable coefficients. Numer. Methods Partial Differ. Equ. Int. J. 2001, 17, 619-631. [CrossRef]

10. Pimenov, V.G.; Hendy, A.S.; De Staelen, R.H. On a class of non-linear delay distributed order fractional diffusion equations. J. Comput. Appl. Math. 2017, 318, 433-443. [CrossRef]

11. Hendy, A.S.; Macías-Díaz, J. A novel discrete Gronwall inequality in the analysis of difference schemes for time-fractional multi-delayed diffusion equations. Commun. Nonlinear Sci. Numer. Simul. 2019, 73, 110-119. [CrossRef]

12. Li, L.; Zhou, B.; Chen, X.; Wang, Z. Convergence and stability of compact finite difference method for nonlinear time fractional reaction-diffusion equations with delay. Appl. Math. Comput. 2018, 337, 144-152. [CrossRef]

13. Hendy, A.S.; Pimenov, V.G.; Macías-Díaz, J.E. Convergence and stability estimates in difference setting for time-fractional parabolic equations with functional delay. Numer. Methods Partial Differ. Equ. 2020, 36, 118-132. [CrossRef]

14. Bhrawy, A.; Zaky, M. Numerical simulation of multi-dimensional distributed-order generalized Schrödinger equations. Nonlinear Dyn. 2017, 89, 1415-1432. [CrossRef] 
15. Hendy, A.S.; Zaky, M.A. Global consistency analysis of L1-Galerkin spectral schemes for coupled nonlinear space-time fractional Schrödinger equations. Appl. Numer. Math. 2020, 156, 276-302. [CrossRef]

16. Zaky, M.; Doha, E.; Tenreiro Machado, J. A spectral numerical method for solving distributed-order fractional initial value problems. J. Comput. Nonlinear Dyn. 2018, 13, 101007. [CrossRef]

17. Zaky, M.A.; Machado, J.T. Multi-dimensional spectral tau methods for distributed-order fractional diffusion equations. Comput. Math. Appl. 2020, 79, 476-488. [CrossRef]

18. Zheng, R.; Liu, F.; Jiang, X.; Turner, I.W. Finite difference/spectral methods for the two-dimensional distributed-order time-fractional cable equation. Comput. Math. Appl. 2020, 80, 1523-1537. [CrossRef]

19. Zhang, Q.; Ren, Y.; Lin, X.; Xu, Y. Uniform convergence of compact and BDF methods for the space fractional semilinear delay reaction-diffusion equations. Appl. Math. Comput. 2019, 358, 91-110. [CrossRef]

20. Çelik, C.; Duman, M. Crank-Nicolson method for the fractional diffusion equation with the Riesz fractional derivative. J. Comput. Phys. 2012, 231, 1743-1750. [CrossRef]

21. Zhao, X.; Sun, Z.Z.; Hao, Z.P. A fourth-order compact ADI scheme for two-dimensional nonlinear space fractional Schrodinger equation. SIAM J. Sci. Comput. 2014, 36, A2865-A2886. [CrossRef]

22. Zhang, Q.; Liu, L.; Zhang, C. Compact scheme for fractional diffusion-wave equation with spatial variable coefficient and delays. Appl. Anal. 2020,1-22. [CrossRef]

23. Zhang, Q.; Zhang, C. A new linearized compact multisplitting scheme for the nonlinear convection-reactiondiffusion equations with delay. Commun. Nonlinear Sci. Numer. Simul. 2013, 18, 3278-3288. [CrossRef]

24. Sun, H.; Sun, Z.Z.; Gao, G.H. Some temporal second order difference schemes for fractional wave equations. Numer. Methods Partial Differ. Equ. 2016, 32, 970-1001. [CrossRef]

25. Gao, G.H.; Sun, Z.Z. A compact finite difference scheme for the fractional sub-diffusion equations. J. Comput. Phys. 2011, 230, 586-595. [CrossRef]

26. Zhao, X.; Sun, Z.Z. Compact Crank-Nicolson schemes for a class of fractional Cattaneo equation in inhomogeneous medium. J. Sci. Comput. 2015, 62, 747-771. [CrossRef]

(C) 2020 by the authors. Licensee MDPI, Basel, Switzerland. This article is an open access article distributed under the terms and conditions of the Creative Commons Attribution (CC BY) license (http:/ / creativecommons.org/licenses/by/4.0/). 